\title{
Non-symmetric isogeometric FEM-BEM couplings
}

\author{
Mehdi Elasmi $^{1}$ (D) Christoph Erath $^{2} \cdot$ Stefan Kurz $^{1}$
}

Received: 17 July 2020 / Accepted: 22 July 2021 / Published online: 19 August 2021

(C) The Author(s) 2021

\begin{abstract}
We present a coupling of the Finite Element and the Boundary Element Method in an isogeometric framework to approximate either two-dimensional Laplace interface problems or boundary value problems consisting of two disjoint domains. We consider the Finite Element Method in the bounded domains to simulate possibly non-linear materials. The Boundary Element Method is applied in unbounded or thin domains where the material behavior is linear. The isogeometric framework allows to combine different design and analysis tools: first, we consider the same type of NURBS parameterizations for an exact geometry representation and second, we use the numerical analysis for the Galerkin approximation. Moreover, it facilitates to perform $h$ - and $p$-refinements. For the sake of analysis, we consider the framework of strongly monotone and Lipschitz continuous operators to ensure well-posedness of the coupled system. Furthermore, we provide a priori error estimates. We additionally show an improved convergence behavior for the errors in functionals of the solution that may double the rate under certain assumptions. Numerical examples conclude the work which illustrate the theoretical results.
\end{abstract}

Keywords Finite element method · Boundary element method · Non-symmetric coupling · Isogeometric analysis · Non-linear operators · Laplacian interface problem · Boundary value problems · Multiple domains · Well-posedness · a priori estimate $\cdot$ Super-convergence $\cdot$ Electromagnetics $\cdot$ Electric machines

Mathematics Subject Classification (2010) $65 \mathrm{~N} 12 \cdot 65 \mathrm{~N} 30 \cdot 65 \mathrm{~N} 38 \cdot 78 \mathrm{M} 10 \cdot 78 \mathrm{M} 15$

\section{Introduction and preliminaries}

In the last decades, simulation gained more and more importance as a fourth pillar of sciences besides theory, experiments, and observations. A successful simulation

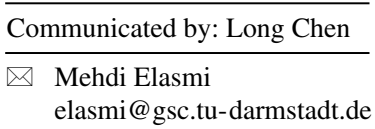

Extended author information available on the last page of the article. 
means a good imitation of some phenomena. This allows the analysis, optimization, and predictions to be made ad hoc with a certain reliability, which depends on the application. For example, this can be achieved for problems that are formulated as boundary and initial value problems by choosing the right mathematical model, a good representation of the computational domain, and a suitable numerical method.

We encounter in this work two types of model problems. First, we consider a Laplacian interface problem in Section 2. Its specificity lies in the combination of a possibly non-linear and non-homogeneous problem in a bounded domain with a linear and homogeneous problem in an unbounded domain. Formally, we aim to find the solution $u$ in the interior domain $\Omega$, such that

$$
-\operatorname{div}(\mathcal{U} \nabla u)=f
$$

where $\mathcal{U}$ is a given non-linear diffusion tensor and $f$ the given right-hand side. In the exterior domain $\mathbb{R}^{2} \backslash \bar{\Omega}$ we are looking for a solution $u^{\mathrm{e}}$ which satisfies

$$
-\Delta u^{\mathrm{e}}=0 \text {. }
$$

Both solutions are connected through suitable jump conditions on the boundary $\partial \Omega$, namely,

$$
\begin{aligned}
u_{\mid \partial \Omega}-u_{\mid \partial \Omega}^{\mathrm{e}} & =u_{0}, \\
\mathcal{U} \nabla u_{\mid \partial \Omega} \cdot v-\nabla u^{\mathrm{e}}{ }_{\mid \partial \Omega} \cdot v & =\phi_{0},
\end{aligned}
$$

where $v$ is an outer normal vector with respect to $\Omega, u_{0}$ and $\phi_{0}$ are given data. Furthermore, the following decay condition for $|x| \rightarrow \infty$ is needed

$$
u^{\mathrm{e}}=\mathcal{O}\left(|x|^{-1}\right)
$$

Note that an additional compatibility condition will be required to ensure the last equation. This will be addressed in the next section. This type of model describes a wide class of engineering and physical applications. For instance, one example for linear elasticity can be found in [43]. We address the second type of model in Section 4. It is a Boundary Value Problem (BVP) with two disjoint domains $\Omega_{1}$ and $\Omega_{2}$, which are separated by a (thin) gap, which we call $\Omega_{\mathrm{b}}$. In the domains we allow non-linear equations. However, the gap is assumed to be filled with a linear material, where the simplest form is air. For a visualization we refer to Fig. 1. The resulting BVP is similar to the one introduced above, however, we prescribe here in addition homogeneous Dirichlet boundary conditions on the interior boundary of $\Omega_{1}$ and on the exterior one of $\Omega_{2}$, which we denote by $\partial \Omega_{0,1}$ and $\partial \Omega_{0,2}$, respectively. Moreover, we remove the decay condition, since the domain $\Omega_{\mathrm{b}}$ is also bounded. Altogether, we consider the following BVP: Find $u_{1}, u_{2}$ and $u_{\mathrm{b}}$ such that

$$
\begin{aligned}
-\operatorname{div}\left(\mathcal{U}_{i} \nabla u_{i}\right) & =f_{i} & & \text { in } \Omega_{i}, \\
-\Delta u_{\mathrm{b}} & =0 & & \text { in } \Omega_{\mathrm{b}}, \\
u_{\mathrm{b} \mid \partial \Omega_{i}}-u_{i \mid \partial \Omega_{i}} & =u_{0, i} & & \text { on } \partial \Omega_{i} \backslash \partial \Omega_{0, i}, \\
\mathcal{U}_{i} \nabla u_{i \mid \partial \Omega_{i}} \cdot v_{i}+\nabla u_{\mathrm{b} \mid \partial \Omega_{i}} \cdot v_{\mathrm{b}} & =\phi_{0, i} & & \text { on } \partial \Omega_{i} \backslash \partial \Omega_{0, i}, \\
u_{i \mid \partial \Omega_{0, i}} & =0 & & \text { on } \partial \Omega_{0, i},
\end{aligned}
$$


where $i=1,2$. Here, $\mathcal{U}_{i}$ are two non-linear diffusion tensors, $f_{i}, u_{0, i}$, and $\phi_{0, i}$ are given data, $v_{i}$ and $v_{\mathrm{b}}$ corresponding normal vectors. This model is particularly used for the simulation of electro-mechanical energy converters. An example is an electric machine discussed in [6]. In general, the air gap is very thin. The other two domains are modeled separately for a facilitation of a possible rotation of the interior part, called rotor in case of an electric machine. This movement is induced by the interaction of electromagnetic fields in the air gap. The computation of forces and torques are therefore one central goal in this type of simulations. Formally, this can be achieved by using the so called Maxwell Stress Tensor (MST) method (see, e.g., [27]). For this, the solution in the air gap as well as its derivatives are needed. These aspects have to be kept in mind for a suitable choice of a numerical method.

The coupling of the Finite Element Method (FEM) and the Boundary Element Methods (BEM) appears to be an intuitive and straightforward choice for the above described problems. Indeed, the FEM is well established and widely used for possibly non-linear problems in bounded domains. On the other side the BEM relies on the transfer of the model problem to an integral representation. Further steps then lead to a Galerkin discretization problem on its boundary with certain integral operators. In a post-processing step, a solution can be found in every point of the underlying domain. Hence, BEM is suitable to handle problems with an unbounded domain where we do not have to truncate the domain since the discretization itself is done on the boundary. We remark that a truncation would be mandatory if we would apply FEM. Since the BEM discretization takes place on a boundary of the domain, it is also very attractive to get a solution in the thin gap described above. For a mesh-based method in the whole domain, e.g., like FEM, it is very difficult to find a mesh for such a thin gap, where the numerical method remains stable. The discretization on the boundary with BEM and the post-processing afterwards avoids this problem. However, to apply the BEM we need to know the fundamental solution of the underlying problem. Note that this restricts the application of BEM especially for non-linear problems. Therefore, we apply BEM in this work for two different applications: first in the exterior unbounded domain and second in the thin air gap. In both cases we consider the Laplace operator for the BEM part, where the fundamental solution can be given explicitly.

In the literature, we distinguish several types of FEM-BEM coupling techniques. These coupling procedures differ solely in the considered representation of the Boundary Integral Equations (BIE), which are the basis for BEM. In order to introduce briefly the considered BIEs, we envisage first the following Laplace equation

$$
-\Delta u=0 \quad \text { in } \Omega^{\kappa}, \quad \kappa=0,1,
$$

where $\Omega^{0} \subseteq \mathbb{R}^{2}$ is a bounded domain with Lipschitz boundary $\Gamma$, and $\Omega^{1}=\mathbb{R}^{2} \backslash \overline{\Omega^{0}}$ is the corresponding unbounded domain. Hence, $\Gamma=\overline{\Omega^{0}} \cap \overline{\Omega^{1}}$. Note that (3) is an interior problem for $\kappa=0$ and an exterior problem for $\kappa=1$. In the latter case, we additionally assume the decay condition $u(x)=C_{\infty} \log |x|+\mathcal{O}(1 /|x|)$ for $|x| \rightarrow \infty$ with the unknown constant $C_{\infty}$ (see also Remark 1). For some $x \in \Omega^{\kappa}$, the solution $u(x)$ is given by the representation formula

$$
u(x)=(-1)^{\kappa}\left(\int_{\Gamma} G(x, y) \phi(y) \mathrm{d} \sigma_{y}-\int_{\Gamma} \partial_{\nu(y)} G(x, y) u_{\mid \Gamma}(y) \mathrm{d} \sigma_{y}\right),
$$


where $G(x, y)=-\frac{1}{2 \pi} \log |x-y|$ denotes the fundamental solution of the Laplace operator, $v(y)$ is an outer normal vector on $\Gamma$ pointing outward with respect to $\Omega^{0}$ at $y \in \Gamma$, and $\left(u_{\mid \Gamma}, \phi:=\partial_{\nu} u_{\mid \Gamma}\right)$ are the unknown or partially unknown Cauchy data. Hereby, the notation $u_{\mid \Gamma}$ means the trace of $u$ with respect to $\Gamma$. Note that we omit to write the trace operators in this work due to readability. Taking the trace of the representation formula yields the BIE

$$
u_{\mid \Gamma}=(-1)^{\kappa}\left(\left(\frac{(-1)^{\kappa}}{2}-\mathcal{K}\right) u_{\mid \Gamma}+\mathcal{V} \phi\right) .
$$

We refer to [41, Chapter 7] for more details. The invoked Boundary Integral Operators $(\mathrm{BIO})$, the single layer operator $\mathcal{V}$ and the double layer operator $\mathcal{K}$, are given for smooth enough inputs by

$$
(\mathcal{V} \phi)(x)=\int_{\Gamma} G(x, y) \phi(y) \mathrm{d} \sigma_{y} \quad \text { and } \quad\left(\mathcal{K} u_{\mid \Gamma}\right)(x)=\int_{\Gamma} \partial_{\nu(y)} G(x, y) u_{\mid \Gamma}(y) \mathrm{d} \sigma_{y} .
$$

They can be extended continuously to linear and bounded operators such that

$$
\mathcal{V}: H^{s-\frac{1}{2}}(\Gamma) \rightarrow H^{s+\frac{1}{2}}(\Gamma) \quad \text { and } \quad \mathcal{K}: H^{s+\frac{1}{2}}(\Gamma) \rightarrow H^{s+\frac{1}{2}}(\Gamma)
$$

for $s \in\left[-\frac{1}{2}, \frac{1}{2}\right]$, c.f. [9, Theorem 1]. In particular, the boundary integral operator $\mathcal{V}$ is additionally symmetric, and $H^{-\frac{1}{2}}(\Gamma)$-elliptic, if $\operatorname{diam}(\Omega)<1$ (see, e.g., [41, Theorem 6.23]). The properties of $\mathcal{V}$ induce the norm equivalence

$$
\|\psi\|_{\mathcal{V}}^{2}:=\langle\psi, \mathcal{V} \psi\rangle_{\Gamma} \simeq\|\psi\|_{H^{-\frac{1}{2}(\Gamma)}}^{2}
$$

In the previous lines, the mentioned spaces have to be understood as follows: for $k>0, H^{k}(\cdot)$ denotes the standard Sobolev space equipped with the usual norm $\|\cdot\|_{H^{k}(\cdot)}$. Moreover, the space $H^{k-\frac{1}{2}}(\Gamma)$ is the trace space of $H^{k}(\Omega)$. The natural duality pairings $\langle\cdot, \cdot\rangle_{\Omega}$ and $\langle\cdot, \cdot\rangle_{\Gamma}$ are obtained by the extended $L^{2}$-scalar products $(\cdot, \cdot)_{\Omega}$ of $\Omega$ and $(\cdot, \cdot)_{\Gamma}$ of $\Gamma$, respectively. Spaces with negative exponents $H^{-k}(\Gamma)$ are defined as dual spaces of $H^{k}(\Gamma)$ with respect to the duality pairing induced by $L^{2}$. Furthermore, for the unbounded domain $\Omega^{1}$ we need functions with local behavior. We denote them by $H_{\text {loc }}^{1}\left(\Omega^{1}\right):=\left\{v: \Omega^{1} \rightarrow \mathbb{R} \mid v_{\mid K} \in H^{1}(K)\right.$ for all $K \subset$ $\overline{\Omega^{1}}$ compact\}. Finally, we write $H^{1}(\Omega)^{\prime}$ for the dual space of $H^{1}(\Omega)$. We recall that

$$
\left\langle\psi, v_{\mid \Gamma}\right\rangle_{\Gamma} \leq\|\psi\|_{H^{-\frac{1}{2}(\Gamma)}}\left\|v_{\mid \Gamma}\right\|_{H^{\frac{1}{2}(\Gamma)}} \leq C_{\text {tr }}\|\psi\|_{H^{-\frac{1}{2}(\Gamma)}}\|v\|_{H^{1}(\Omega)}
$$

holds for all $v \in H^{1}(\Omega)$ and $\psi \in H^{-\frac{1}{2}}(\Gamma)$, where the trace inequality is encoded with the trace constant $C_{\mathrm{tr}}>0$.

In the following, we describe a variational ansatz to get a weak form of the model problem. As mentioned above, there are several coupling strategies possible. If we describe the FEM part by the weak form of the well-known Green's first formula, a coupling with the weak form of $(5)(\kappa=1)$ leads to the so called Johnson-Nédélec coupling introduced in [25]. The combined weak form is non-symmetric even though the model problem itself is symmetric. Also a Galerkin discretization leads to a non-symmetric system of linear equations. Therefore, this coupling is also known as non-symmetric coupling, where the unknowns are the $u$ of the FEM part and the 
conormal derivate $\phi$ of the BEM part. To symmetrize this system in case of a symmetric model problem, we first observe that taking the conormal derivative of (4) leads to another integral equation with two other integral operators. A modification of the Johnson-Nédélec coupling with this additional integral equation renders the coupled problem symmetric. This procedure appeared first in [10] and is known as Costabel's symmetric coupling. The price of the symmetry is the use of four BIOs, which is computationally more expensive. However, there are still only two unknowns involved. A coupling method with three unknowns, i.e., additionally the trace $u_{\mid \Gamma}$ of the BEM part is an unknown, is called a three field coupling [14]. A coupling procedure with the so called indirect ansatz is also possible and called Bielak-MacCamy coupling [5]. With this strategy, however, one unknown of the BEM part has no physical meaning.

Because of the advantages of the non-symmetric coupling, we consider in this work only this type of coupling. We will introduce it formally in Section 2. For a long time mathematical analysis for this coupling was only available for smooth boundaries due to the use of a compactness argument of the double layer operator $\mathcal{K}$ [25]. In particular, Lipschitz boundaries were excluded . However, a decade ago Sayas [38] provided in fact the first analysis also for Lipschitz boundaries. This work influenced several variations and improvements, e.g., $[2,15,42]$ to mention a few but not all. Hence, the non-symmetric coupling became a more natural choice, especially, if a part of the model problem is non-symmetric or non-linear. In this work, we use the results of [2], where an extension to non-linear interface problems has been addressed and combine this result with the proof shown in [15]. Furthermore, we also use [32], which extended the proofs for the linear interface problem to certain Boundary Value Problems, i.e., to the second type of problem considered here. For the linear interface problem with a general second order problem in the interior domain, we also refer to [15] for a rigorous and, to the authors' knowledge, sharpest ellipticity estimate. Recently, a complete analysis of a parabolic-elliptic interface problem with a full discretization in the sense of a non-symmetric FEM-BEM coupling for spatial discretization was published in [13]. Note that such a system arises, for instance, in the modeling of eddy currents in the magneto-quasi-static regime [28].

Now, having described the weak form of the model problem with the proposed FEM and BEM parts, we still need to take two major decisions for a successful simulation: a suitable discretization technique, i.e., choosing concrete ansatz spaces for the FEM and BEM, and a good representation of the geometry. These steps are typically made independently, which complicates meshing and remeshing procedures without altering the original geometry. In order to circumvent this, design step and numerical analysis can be combined by considering the same type of basis functions. Hence the geometrical modeling is also used to design ansatz functions in the Galerkin discretization schemes for the approximation of the solution. Such a method is proposed in [24]. It is based on using Non-Uniform Rational B-Splines (NURBS) for the unification of Computer Aided Design (CAD) and Finite Element Analysis (FEA). This method is called IsoGeometric Analysis (IGA). The first isogeometric BEM simulation of collocation type can be found in [35, 40]. Moreover, fast methods for isogeometric BEM have been successfully implemented in [12, 23, 29], which reduces the known high computational complexity of such an application due to the dense matrices produced by the BEM. This makes the method more 
attractive even for more realistic and complex applications (see, e.g., [8] and [6]). A rigorous mathematical analysis for isogemetric FEM started in [4, 44] and for isogemetric Galerkin BEM in [18-22]. For our purpose the results [7, 44] together with [2, 15,32 ] play a central role in proving the validity and an a priori error estimate of the FEM-BEM coupling in the isogeometric context, which is done in this manuscript for the first time.

The rest of this paper is organized as follows: in Section 2, the non-linear interface problem is addressed. We consider the framework of Lipschitz continuous and strongly monotone operators such as given in [46] and used in [2]. Strong monotonicity of the non-symmetric weak form is shown analogously to the arguments in [15] by adapting the setting to non-linear operators. Moreover, well-posedness of the coupling is stated. Section 3 is devoted to the Galerkin discretization of the non-symmetric coupling. Thereby, we introduce the isogeometric framework and the necessary discrete spaces. We derive some error estimates for the conforming isogeometric discretization. In Section 4, we extend the model to a Boundary Value Problem. More precisely, the model domain is split in two disjoint domains, which are separated by a thin (air) gap. First, a variational formulation of the coupled problem is derived. Then we show well-posedness and stability of the method. Furthermore, we discuss a super-convergence result for the evaluation of the solution in the BEM domain. In the last Section 5, we confirm the theoretical results by conducting one numerical example for each model problem. The work is completed by some conclusions and an outlook.

\section{Interface problem}

Let $\Omega \subset \mathbb{R}^{2}$ be a bounded domain with Lipschitz boundary $\Gamma=\partial \Omega$ and $\Omega^{\mathrm{e}}:=$ $\mathbb{R}^{2} \backslash \bar{\Omega}$ the corresponding unbounded (exterior) domain. Furthermore, to guarantee the $H^{-\frac{1}{2}}(\Gamma)$-ellipticity of the boundary integral operator $\mathcal{V}$, we assume diam $(\Omega)<$ 1. This assumption can merely be achieved by scaling. We consider the following interface problem: Find $\left(u, u^{\mathrm{e}}\right) \in H^{1}(\Omega) \times H_{\mathrm{loc}}^{1}\left(\Omega^{\mathrm{e}}\right)$ such that

$$
\begin{aligned}
-\operatorname{div}(\mathcal{U} \nabla u) & =f & & \text { in } \Omega, \\
-\Delta u^{\mathrm{e}} & =0 & & \text { in } \Omega^{\mathrm{e},} \\
u_{\mid \Gamma}-u^{\mathrm{e}}{ }_{\mid \Gamma} & =u_{0} & & \text { on } \Gamma, \\
\mathcal{U} \nabla u_{\mid \Gamma} \cdot v-\nabla u_{\mid \Gamma}^{\mathrm{e}_{\mid} \cdot v} & =\phi_{0} & & \text { on } \Gamma, \\
u^{\mathrm{e}} & =\mathcal{O}\left(|x|^{-1}\right) & & \text { for }|x| \rightarrow \infty .
\end{aligned}
$$

We remind that $v$ denotes the outer normal vector with respect to $\Omega$ and $\mathcal{U}: \mathbb{R}^{2} \rightarrow$ $\mathbb{R}^{2}$ is a possibly non-linear diffusion tensor. The right-hand side is given by $f \in$ $H^{1}(\Omega)^{\prime}, u_{0} \in H^{\frac{1}{2}}(\Gamma)$ is the jump in the Dirichlet data, and $\phi_{0} \in H^{-\frac{1}{2}}(\Gamma)$ the jump in the Neumann data.

To ensure the right decay condition (9e) at infinity, we have to assume the additional condition

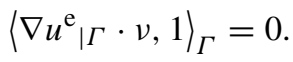


This can be transformed into a compatibility condition on the data, i.e.,

$$
\langle f, 1\rangle_{\Omega}+\left\langle\phi_{0}, 1\right\rangle_{\Gamma}=0
$$

Remark 1 Note that the assumption to ensure the decay condition is only needed in the two-dimensional case. Alternatively, (9e) can be replaced by a logarithmic decay of the solution in two dimensions to avoid the additional assumption on $\nabla u^{\mathrm{e}}{ }_{\mid \Gamma}$, i.e.,

$$
u^{\mathrm{e}}=C \log |x|+\mathcal{O}\left(|x|^{-1}\right) \quad \text { for }|x| \rightarrow \infty
$$

with $C:=\frac{1}{2 \pi}\left\langle\nabla u_{\mid \Gamma}^{\mathrm{e}} \cdot v, 1\right\rangle_{\Gamma}$ or equivalently $C:=-\frac{1}{2 \pi}\left(\langle f, 1\rangle_{\Omega}+\left\langle\phi_{0}, 1\right\rangle_{\Gamma}\right)$, which can be easily verified in the weak formulation below.

As mentioned above, the diffusion tensor $\mathcal{U}$ can be a non-linear operator. To apply standard theory for non-linear operators (see, e.g., [46]), we assume throughout the manuscript that $\mathcal{U}$ is Lipschitz continuous and strongly monotone:

(A1) Lipschitz continuity:

$$
\exists C_{\text {Lip }}^{\mathcal{U}}>0 \text { such that }|\mathcal{U} x-\mathcal{U} y| \leq C_{\text {Lip }}^{\mathcal{U}}|x-y|, \forall x, y \in \mathbb{R}^{2} .
$$

(A2) strong monotonicity:

$$
\exists C_{\text {ell }}^{\mathcal{U}}>0 \text { such that }(\mathcal{U} \nabla u-\mathcal{U} \nabla v, \nabla u-\nabla v)_{\Omega} \geq C_{\text {ell }}^{\mathcal{U}}\|\nabla u-\nabla v\|_{L^{2}(\Omega)}^{2}, \forall u, v \in H^{1}(\Omega) .
$$

The derivation of a non-symmetric variational form follows a standard procedure: In the variational form of (9a), we replace the Neumann data by the jump condition (9d) to couple the interior problem with the conormal derivative with $\phi:=\partial_{\nu} u_{\mid \Gamma}^{\mathrm{e}}=$ $\nabla u_{\mid \Gamma}^{\mathrm{e}} \cdot v$ of the exterior problem. For the second equation we use the exterior integral (5) with $\kappa=1$, and insert the jump condition (9c) to couple this with the interior trace.

Hence, the weak formulation of the non-symmetric coupling problem (9) reads: Find $\mathbf{u}:=(u, \phi) \in H^{1}(\Omega) \times H^{-\frac{1}{2}}(\Gamma)$ such that

$$
\begin{aligned}
(\mathcal{U} \nabla u, \nabla v)_{\Omega}-\left\langle\phi, v_{\mid \Gamma}\right\rangle_{\Gamma} & =\langle f, v\rangle_{\Omega}+\left\langle\phi_{0}, v_{\mid \Gamma}\right\rangle_{\Gamma}, \\
\left\langle\psi,\left(\frac{1}{2}-\mathcal{K}\right) u_{\mid \Gamma}\right\rangle_{\Gamma}+\langle\psi, \mathcal{V} \phi\rangle_{\Gamma} & =\left\langle\psi,\left(\frac{1}{2}-\mathcal{K}\right) u_{0}\right\rangle_{\Gamma}
\end{aligned}
$$

hold $\forall \mathbf{v}:=(v, \psi) \in H^{1}(\Omega) \times H^{-\frac{1}{2}}(\Gamma)$.

This variational form can be written in a compact form. For this we introduce a product space with corresponding norm, i.e.,

$$
\begin{aligned}
\mathcal{H} & :=H^{1}(\Omega) \times H^{-\frac{1}{2}}(\Gamma), \quad\|\mathbf{v}\|_{\mathcal{H}}:=\left(\|v\|_{H^{1}(\Omega)}^{2}+\|\psi\|_{H^{-\frac{1}{2}}(\Gamma)}^{2}\right)^{\frac{1}{2}} \\
\text { for } \mathbf{v} & :=(v, \psi) \in \mathcal{H} .
\end{aligned}
$$


Problem 1 Find $\mathbf{u} \in \mathcal{H}:=H^{1}(\Omega) \times H^{-\frac{1}{2}}(\Gamma)$ such that $a(\mathbf{u}, \mathbf{v})=\ell(\mathbf{v})$ holds $\forall \mathbf{v} \in \mathcal{H}$ with the linear form (linear in the second argument) $a: \mathcal{H} \times \mathcal{H} \rightarrow \mathbb{R}$,

$$
a(\mathbf{u}, \mathbf{v}):=(\mathcal{U} \nabla u, \nabla v)_{\Omega}-\left\langle\phi, v_{\mid \Gamma}\right\rangle_{\Gamma}+\left\langle\psi,\left(\frac{1}{2}-\mathcal{K}\right) u_{\mid \Gamma}\right\rangle_{\Gamma}+\langle\psi, \mathcal{V} \phi\rangle_{\Gamma},
$$

and the linear functional $\ell$ on $\mathcal{H}$,

$$
\ell(\mathbf{v}):=\langle f, v\rangle_{\Omega}+\left\langle\phi_{0}, v_{\mid \Gamma}\right\rangle_{\Gamma}+\left\langle\psi,\left(\frac{1}{2}-\mathcal{K}\right) u_{0}\right\rangle_{\Gamma} .
$$

It is easy to check that $a(\mathbf{v}, \mathbf{v})$ is not elliptic, e.g., insert $\mathbf{v}=(1,0)$. Hence, [2] suggested an implicit stabilization where the stabilized problem is equivalent to the original one, i.e., a solution of the original problem is also a solution of the stabilized one and vice versa. Thus, the analysis is done with the aid of the stabilized form, i.e., well-posedness is inherited to the original problem. For implementation purposes, we still use the original problem. The stabilized problem reads:

Problem 2 Find $\mathbf{u} \in \mathcal{H}$ such that $\widetilde{a}(\mathbf{u}, \mathbf{v})=\tilde{\ell}(\mathbf{v})$ holds $\forall \mathbf{v} \in \mathcal{H}$, where we define with

$$
s(\mathbf{v}):=\left\langle 1,\left(\frac{1}{2}-\mathcal{K}\right) v_{\mid \Gamma}\right\rangle_{\Gamma}+\langle 1, \mathcal{V} \psi\rangle_{\Gamma}, \quad \mathbf{v}:=(v, \psi)
$$

the stabilized linear form

$$
\widetilde{a}(\mathbf{u}, \mathbf{v}):=a(\mathbf{u}, \mathbf{v})+s(\mathbf{u}) s(\mathbf{v}),
$$

and the functional

$$
\tilde{\ell}(\mathbf{v}):=\ell(\mathbf{v})+\left\langle 1,\left(\frac{1}{2}-\mathcal{K}\right) u_{0}\right\rangle_{\Gamma} s(\mathbf{v}) .
$$

Lemma 1 [2] The original and the stabilized formulation are equivalent, i.e., $\mathbf{u} \in \mathcal{H}$ solves Problem 1 if and only if it solves Problem 2, and vice versa.

In order to state well-posedness for Problem 2, and thanks to Lemma 1 also for Problem 1, we follow standard results for monotone operators [46]. First, we note that the form $\tilde{a}(\mathbf{u}, \mathbf{v})$ induces a non-linear operator $\widetilde{\mathcal{A}}: \mathcal{H} \rightarrow \mathcal{H}^{\prime}$ by

$$
\langle\widetilde{\mathcal{A}}(\mathbf{u}), \mathbf{v}\rangle:=\widetilde{a}(\mathbf{u}, \mathbf{v}), \quad \forall \mathbf{u}, \mathbf{v} \in \mathcal{H}
$$

where $\mathcal{H}^{\prime}$ denotes the dual space of $\mathcal{H}$. This allows us to prove the following lemma.

Theorem 1 [2, 15] Let us consider the non-linear operator $\widetilde{\mathcal{A}}: \mathcal{H} \rightarrow \mathcal{H}^{\prime}$ defined in (13) with $\mathcal{H}=H^{1}(\Omega) \times H^{-\frac{1}{2}}(\Gamma)$. We state the following three assertions.

1. $\tilde{\mathcal{A}}$ is Lipschitz continuous, i.e., there exists $C_{\mathrm{Lip}}>0$ such that

$$
\|\widetilde{\mathcal{A}}(\mathbf{u})-\tilde{\mathcal{A}}(\mathbf{v})\|_{\mathcal{H}^{\prime}} \leq C_{\text {Lip }}\|\mathbf{u}-\mathbf{v}\|_{\mathcal{H}}
$$

for all $\mathbf{u}, \mathbf{v} \in \mathcal{H}$. 
2. if $C_{\mathrm{ell}}^{\mathcal{U}}>\frac{1}{4}$ then

$$
\langle\widetilde{\mathcal{A}}(\mathbf{u})-\widetilde{\mathcal{A}}(\mathbf{v}), \mathbf{u}-\mathbf{v}\rangle \geq C_{\text {stab }}\left(\|\nabla u-\nabla v\|_{L^{2}(\Omega)}^{2}+\|\phi-\psi\|_{\mathcal{V}}^{2}+s(\mathbf{u}-\mathbf{v})^{2}\right)
$$

for all $\mathbf{u}:=(u, \phi) \in \mathcal{H}, \mathbf{v}:=(v, \psi) \in \mathcal{H}$ with the norm $\|\psi\|_{\mathcal{V}}^{2}:=\langle\psi, \mathcal{V} \psi\rangle_{\Gamma}$ and with

$$
C_{\text {stab }}=\min \left\{1, \frac{1}{2}\left(1+C_{\mathrm{ell}}^{\mathcal{U}}-\sqrt{\left(C_{\mathrm{ell}}^{\mathcal{U}}-1\right)^{2}+1}\right)\right\} .
$$

3. if $C_{\mathrm{ell}}^{\mathcal{U}}>\frac{1}{4}$ then $\widetilde{\mathcal{A}}$ is strongly monotone, i.e., there exists $C_{\mathrm{ell}}>0$ such that

$$
\langle\widetilde{\mathcal{A}}(\mathbf{u})-\widetilde{\mathcal{A}}(\mathbf{v}), \mathbf{u}-\mathbf{v}\rangle \geq C_{\text {ell }}\|\mathbf{u}-\mathbf{v}\|_{\mathcal{H}}^{2}
$$

for all $\mathbf{u}, \mathbf{v} \in \mathcal{H}$.

Remark 2 The constants $C_{\text {Lip }}$ and $C_{\text {ell }}$ in Theorem 1 depend on various other constants, namely, the continuity constants of the boundary integral operators $\mathcal{V}$ and $\mathcal{K}$, the ellipticity constant of $\mathcal{V}, C_{\text {Lip }}^{\mathcal{U}}, C_{\text {ell }}^{\mathcal{U}}$ and $C_{\text {tr }}$, in particular from $\Omega$ and $\Gamma$.

Proof of Theorem 1 The Lipschitz continuity of $\tilde{\mathcal{A}}$ follows from the Lipschitz continuity of $\mathcal{U}$, and the continuity of the integral operators.

The proof of the second assertion follows the lines of [15, Theorem 1] for $\beta=1$. We replace the coercivity estimate of the bilinear form $(\mathcal{U} \nabla u, \nabla v)_{\Omega}$ considered in [15] for a linear $\mathcal{U}$ by the strong monotonicity property of $\mathcal{U}$, i.e,

$$
(\mathcal{U} \nabla u-\mathcal{U} \nabla v, \nabla u-\nabla v)_{\Omega} \geq C_{\mathrm{ell}}^{\mathcal{U}}\|\nabla u-\nabla v\|_{L^{2}(\Omega)}^{2} .
$$

The restriction $C_{\mathrm{ell}}^{\mathcal{U}}>\frac{1}{4}$ is a direct result of the use of a contractivity result for the double layer operator $\mathcal{K}$ (see [31, Lemma 2.1]) with a constant $C_{\mathcal{K}} \in\left[\frac{1}{2}, 1\right.$ ), where we use the worst case of $C_{\mathcal{K}}=1$ in the statement.

For the last assertion we note the norm equivalence (7), and by a Rellich compactness argument it can be shown [2, Lemma 10] that

$$
\|\mathbf{v}\|^{2}:=\|\nabla v\|_{L^{2}(\Omega)}^{2}+\|\psi\|_{\mathcal{V}}^{2}+s(\mathbf{v})^{2}
$$

defines an equivalent norm in $\mathcal{H}$ for some $\mathbf{v}:=(v, \psi) \in \mathcal{H}$. Together with (14) this leads to the last assertion with $C_{\mathrm{ell}}^{\mathcal{U}}>\frac{1}{4}$, which is required for (14).

The following theorem follows directly from the theoretical result [46, Theorem 25.B].

Theorem 2 (Well-posedness) Provided that $C_{\mathrm{ell}}^{\mathcal{U}}>\frac{1}{4}$, there exists a unique solution $\mathbf{u}:=(u, \phi) \in \mathcal{H}$ of the variational Problem 1 for any $\left(f, u_{0}, \phi_{0}\right) \in H^{1}(\Omega)^{\prime} \times$ $H^{\frac{1}{2}}(\Gamma) \times H^{-\frac{1}{2}}(\Gamma)$.

Proof From Theorem 1 it follows that the induced operator $\widetilde{\mathcal{A}}$ of $\widetilde{a}(\cdot, \cdot)$ is strongly monotone and Lipschitz continuous for $C_{\mathrm{ell}}^{\mathcal{U}}>\frac{1}{4}$. Hence, by using [46, Theorem 25.B], there exists a unique solution $\mathbf{u}:=(u, \phi) \in \mathcal{H}$ of the variational 
Problem 2, for any $\left(f, u_{0}, \phi_{0}\right) \in H^{1}(\Omega)^{\prime} \times H^{\frac{1}{2}}(\Gamma) \times H^{-\frac{1}{2}}(\Gamma)$. Thanks to the equivalence stated in Lemma 1 , this is also the unique solution of Problem 1.

For some engineering applications, where the non-linear operator $\mathcal{U}$ has a special form, we can state the following stabilization result.

Lemma 2 Let $C_{\mathrm{ell}}^{\mathcal{U}}>\frac{1}{4}$ and let the non-linear operator $\mathcal{U}$ be of the form $\mathcal{U} \nabla u:=$ $g(|\nabla u|) \nabla u$ with a non-linear function $g: \mathbb{R} \rightarrow \mathbb{R}$. Then, for the solution $\mathbf{u}:=$ $(u, \phi) \in \mathcal{H}$ of Problem 1, we have the stability result

$$
\|\mathbf{u}\|_{\mathcal{H}} \leq C\left(\|f\|_{H^{1}(\Omega)^{\prime}}+\left\|u_{0}\right\|_{H^{\frac{1}{2}(\Gamma)}}+\left\|\phi_{0}\right\|_{H^{-\frac{1}{2}(\Gamma)}}\right), \quad C>0 .
$$

Proof Let $\mathbf{v} \in \mathcal{H}$ be arbitrary. We know from the strong monotonicity of $\widetilde{\mathcal{A}}$ that

$$
C_{\mathrm{ell}}\|\mathbf{u}-\mathbf{v}\|_{\mathcal{H}}^{2} \leq\langle\tilde{\mathcal{A}}(\mathbf{u})-\tilde{\mathcal{A}}(\mathbf{v}), \mathbf{u}-\mathbf{v}\rangle \text {. }
$$

Without loss of generality, we choose $\mathbf{v}=(0,0)$ and note that $\mathcal{U} \nabla v=0$. Thanks to Lemma $1 \mathbf{u}:=(u, \phi)$ is also the unique solution of the Problem 2. Thus, we conclude that

$$
\begin{aligned}
C_{\mathrm{ell}}\|\mathbf{u}\|_{\mathcal{H}}^{2} & \leq\langle\tilde{\mathcal{A}}(\mathbf{u}), \mathbf{u}\rangle=\tilde{\ell}(\mathbf{u}), \\
& =\langle f, u\rangle_{\Omega}+\left\langle\phi_{0}, u_{\mid \Gamma}\right\rangle_{\Gamma}+\left\langle\phi,\left(\frac{1}{2}-\mathcal{K}\right) u_{0}\right\rangle_{\Gamma}+\left\langle 1,\left(\frac{1}{2}-\mathcal{K}\right) u_{0}\right\rangle_{\Gamma} s(\mathbf{u})
\end{aligned}
$$

with $s(\mathbf{u}):=\left\langle 1,\left(\frac{1}{2}-\mathcal{K}\right) u_{\mid \Gamma}\right\rangle_{\Gamma}+\langle 1, \mathcal{V} \phi\rangle_{\Gamma}$. Next we use the Cauchy-Schwarz inequality (8) along with the boundedness of $\mathcal{K}$ and $\mathcal{V}$. Then, rearranging the terms yields

$$
\begin{aligned}
C_{\mathrm{ell}}\|\mathbf{u}\|_{\mathcal{H}}^{2} \leq & \left(\|f\|_{H^{1}(\Omega)^{\prime}}+C_{\text {tr }}\left\|\phi_{0}\right\|_{H^{-\frac{1}{2}(\Gamma)}}+\|1\|_{H^{-\frac{1}{2}(\Gamma)}}\left(\frac{1}{2}+C^{\mathcal{K}}\right)^{2} C_{\text {tr }}\left\|u_{0}\right\|_{H^{\frac{1}{2}}(\Gamma)}\right)\|u\|_{H^{1}(\Omega)} \\
& +\|1\|_{H^{-\frac{1}{2}(\Gamma)}}\left(\left(\frac{1}{2}+C^{\mathcal{K}}\right)\left(1+C^{\mathcal{V}}\right)\left\|u_{0}\right\|_{H^{\frac{1}{2}}(\Gamma)}\right)\|\phi\|_{H^{-\frac{1}{2}}(\Gamma)},
\end{aligned}
$$

where $C^{\mathcal{K}}, C^{\mathcal{V}}>0$ denote the continuity constants of the boundary integral operators $\mathcal{K}$ and $\mathcal{V}$, respectively. From this follows the assertion with a constant $C>0$ that depends on $C^{\mathcal{K}}, C^{\mathcal{V}}, C_{\mathrm{tr}}, C_{\mathrm{ell}}$ and $\Gamma$.

Remark 3 Because of Lemma 1, the results obtained for the stabilized formulation is also valid for the original non-symmetric coupling of Problem 1. Hence, in the next section, we only discretize the original problem using a Galerkin approximation. In fact, the stabilized version is only used for analysis purposes.

\section{Galerkin discretization}

Let $V_{\ell} \subset H^{1}(\Omega)$ and $X_{\ell} \subset H^{-\frac{1}{2}}(\Gamma)$ be some finite dimensional subspaces, where the index $\ell$ expresses a refinement level, e.g., in a sequence of mesh refinements. We assume that: 
(A3) The discrete space $X_{\ell}$ contains the constants, i.e.,

$$
\exists \xi \in \bigcap_{\ell \in \mathbb{N}_{0}} X_{\ell} \text { such that }\langle\xi, 1\rangle_{\Gamma} \neq 0 .
$$

We consider a conforming Galerkin discretization of the Problem 1. Replacing the spaces $H^{1}(\Omega)$ and $H^{-\frac{1}{2}}(\Gamma)$ with $V_{\ell}$ and $X_{\ell}$, respectively, leads to the following discrete problem: Find $\mathbf{u}_{\ell}:=\left(u_{\ell}, \phi_{\ell}\right) \in \mathcal{H}_{\ell}:=V_{\ell} \times X_{\ell}$ such that

$$
\begin{aligned}
\left\langle\mathcal{U} \nabla u_{\ell}, \nabla v_{\ell}\right\rangle_{\Omega}-\left\langle\phi_{\ell}, v_{\ell}\right\rangle_{\Gamma} & =\left\langle f, v_{\ell}\right\rangle_{\Omega}+\left\langle\phi_{0}, v_{\ell}\right\rangle_{\Gamma}, \\
\left\langle\psi_{\ell},\left(\frac{1}{2}-\mathcal{K}\right) u_{\ell}\right\rangle_{\Gamma}+\left\langle\psi_{\ell}, \mathcal{V} \phi_{\ell}\right\rangle_{\Gamma} & =\left\langle\psi_{\ell},\left(\frac{1}{2}-\mathcal{K}\right) u_{0}\right\rangle_{\Gamma}
\end{aligned}
$$

hold $\forall \mathbf{v}_{\ell}:=\left(v_{\ell}, \psi_{\ell}\right) \in \mathcal{H}_{\ell}$.

The compact form in the product space $\mathcal{H}_{\ell}$ reads:

Problem 3 Find $\mathbf{u}_{\ell}:=\left(u_{\ell}, \phi_{\ell}\right) \in \mathcal{H}_{\ell}:=V_{\ell} \times X_{\ell}$ such that $a\left(\mathbf{u}_{\ell}, \mathbf{v}_{\ell}\right)=\ell\left(\mathbf{v}_{\ell}\right)$ holds $\forall \mathbf{v}_{\ell}=\left(v_{\ell}, \psi_{\ell}\right) \in \mathcal{H}_{\ell}$. The linear form $a(\cdot, \cdot)$ and the linear functional $\ell$ are defined in (11) and (12), respectively.

Provided that Assumption (A3) is satisfied, the analysis for Problem 3 is done analogously to the continuous Problem 1 since the discrete spaces are conforming. In other words, all the above results including the introduction of a stabilized form and Lemma 1 also apply for the subspaces. In particular, due to Theorem 2 the discrete solution $\mathbf{u}_{\ell}:=\left(u_{\ell}, \phi_{\ell}\right) \in \mathcal{H}_{\ell}:=V_{\ell} \times X_{\ell}$ of Problem 3 exists and is unique. The following quasi-optimality result in the sense of the Céa-type Lemma is a standard but central result, which will be needed in Section 3.2 for the a priori error estimate of the non-symmetric coupling.

Theorem 3 (Quasi-optimality) Let Assumption (A3) hold, and $C_{\mathrm{ell}}^{\mathcal{U}}>\frac{1}{4}$. Moreover, let $\mathbf{u}:=(u, \phi) \in \mathcal{H}$ be the unique solution of Problem 1 , and $\mathbf{u}_{\ell}:=\left(u_{\ell}, \phi_{\ell}\right) \in \mathcal{H}_{\ell}$ the solution of its discrete counterpart Problem 3. Then

$$
\left\|u-u_{\ell}\right\|_{H^{1}(\Omega)}+\left\|\phi-\phi_{\ell}\right\|_{H^{-\frac{1}{2}}(\Gamma)} \leq C_{C e ́ a} \min _{v_{\ell} \in V_{\ell}, \psi_{\ell} \in X_{\ell}}\left(\left\|u-v_{\ell}\right\|_{H^{1}(\Omega)}+\left\|\phi-\psi_{\ell}\right\|_{H^{-\frac{1}{2}}(\Gamma)}\right)
$$

with $C_{\text {Céa }}=\frac{C_{\mathrm{Lip}}}{C_{\mathrm{ell}}}$.

Proof The assertion follows as a result of the main theorem on strongly monotone operators [46, Corollary 25.7]. That means with $\mathbf{v}_{\ell}:=\left(v_{\ell}, \psi_{\ell}\right)$, thanks to Lemma 1 , the strong monotonicity, Galerkin orthogonality, Cauchy-Schwarz inequality, and the Lipschitz continuity we get

$$
\begin{aligned}
C_{\mathrm{ell}}\left\|\mathbf{u}-\mathbf{u}_{\ell}\right\|_{\mathcal{H}}^{2} & \leq\left\langle\widetilde{\mathcal{A}}(\mathbf{u})-\widetilde{\mathcal{A}}\left(\mathbf{u}_{\ell}\right), \mathbf{u}-\mathbf{u}_{\ell}\right\rangle=\left\langle\widetilde{\mathcal{A}}(\mathbf{u})-\widetilde{\mathcal{A}}\left(\mathbf{u}_{\ell}\right), \mathbf{u}-\mathbf{v}_{\ell}\right\rangle \\
& \leq\left\|\widetilde{\mathcal{A}}(\mathbf{u})-\widetilde{\mathcal{A}}\left(\mathbf{u}_{\ell}\right)\right\|_{\mathcal{H}^{\prime}}\left\|\mathbf{u}-\mathbf{v}_{\ell}\right\|_{\mathcal{H}} \\
& \leq C_{\text {Lip }}\left\|\mathbf{u}-\mathbf{u}_{\ell}\right\|_{\mathcal{H}}\left\|\mathbf{u}-\mathbf{v}_{\ell}\right\|_{\mathcal{H}},
\end{aligned}
$$

where the assertion follows directly. 


\subsection{Isogeometric analysis}

The basis functions that are considered for the geometry design in the isogeometric framework are used as ansatz functions for the Galerkin discretization. These functions are typically B-Splines or some extensions of B-Splines, e.g., NURBS, T-Splines etc.

In the following, we introduce briefly the concept of Isogeometric Analysis and refer to [11] for a more detailed introduction, and to [44] and [7] for a mathematical analysis of IGA in the FEM and BEM context, respectively.

Definition 1 Let $p \in \mathbb{N}$ denote the degree, and $k \in \mathbb{N}$ the number of the B-Spline basis functions, with $k>p$. A knot vector $\Xi:=\left\{\xi_{0}, \ldots, \xi_{k+p}\right\}$ is called $p$-open if

$$
0=\xi_{0}=\cdots=\xi_{p}<\xi_{p+1} \leq \cdots \leq \xi_{k-1}<\xi_{k}=\cdots=\xi_{k+p}=1 .
$$

Associated to the knot vector $\Xi, k$ B-Spline basis functions can be defined recursively for $p \geq 1$ by

$$
b_{i}^{p}(x)=\frac{x-\xi_{i}}{\xi_{i+p}-\xi_{i}} b_{i}^{p-1}(x)+\frac{\xi_{i+p+1}-x}{\xi_{i+p+1}-\xi_{i+1}} b_{i+1}^{p-1}(x)
$$

for all $i=0 \ldots k-1$, starting with piecewise constant basis functions for $p=0$, namely,

$$
b_{i}^{0}(x)= \begin{cases}1 & \text { if } \xi_{i} \leq x \leq \xi_{i+1} \\ 0 & \text { otherwise }\end{cases}
$$

Moreover, we denote by $\mathbb{S}_{p}(\Xi)=\operatorname{span}\left\{\left\{b_{i}^{p}\right\}_{i=0}^{k-1}\right\}$ the space of B-Splines of degree $p$ and dimension $k$ in the parameter domain over the knot vector $\Xi$.

Definition 2 Let $f:[0,1] \rightarrow \gamma \subset \mathbb{R}^{d}$ be a B-Spline mapping defined as

$$
f(x)=\sum_{i=0}^{k-1} c_{i} b_{i}^{p}(x)
$$

with $c_{i} \in \mathbb{R}^{d}$ representing an element of a set of control points. The mapping $f$ describes a one-dimensional curve embedded in a $d$-dimensional Euclidian space and is called a B-Spline curve. Moreover, we call $\gamma$ a patch if the mapping $f$ is regular.

As long as the B-Spline mapping $f$ is regular, we can define B-Spline spaces in the physical domain, i.e., over a patch $\gamma$ by using the following transformation

$$
\iota(f)(u)=u \circ f
$$

namely,

$$
\mathbb{S}_{p}(\gamma)=\left\{v: v=u \circ f^{-1}, u \in \mathbb{S}_{p}(\Xi)\right\}
$$


Definition 3 B-Spline spaces on higher dimensional domains are constructed by using tensor product relationships. For example, in $2 D$, we write with $p_{1}, p_{2} \in \mathbb{N}$ and $k_{1}, k_{2} \in \mathbb{N}$

$$
\mathbb{S}_{p_{1}, p_{2}}\left(\Xi_{1}, \Xi_{2}\right)=\operatorname{span}\left\{\left\{b_{i_{1}}^{p_{1}} b_{i_{2}}^{p_{2}}\right\}_{i_{1}=0, i_{2}=0}^{k_{1}-1, k_{2}-1}\right\}
$$

where $p_{1}$ and $p_{2}$ denote the degrees in each parametric direction, and $k_{1} k_{2}$ is the number of the B-Splines basis functions. A B-Spline surface is thus represented by $\mathbf{f}(x):[0,1]^{2} \rightarrow \omega \subset \mathbb{R}^{d}$ with

$$
\mathbf{f}(x)=\sum_{i_{1}=0}^{k_{1}-1} \sum_{i_{2}=0}^{k_{2}-1} \mathbf{c}_{i_{1}, i_{2}} \cdot b_{i_{1}}^{p_{1}}(x) \cdot b_{i_{2}}^{p_{2}}(x),
$$

where $\mathbf{c}_{i_{1}, i_{2}} \in \mathbb{R}^{d}$ is representing an element of a set of control points. If $f$ is regular, we call $\omega$ a patch.

Equivalently, the two-dimensional B-Spline space in the physical domain is defined over a patch $\omega$ by

$$
\mathbb{S}_{p_{1}, p_{2}}(\omega)=\left\{v: v=u \circ \mathbf{f}^{-1}, u \in \mathbb{S}_{p_{1}, p_{2}}\left(\Xi_{1}, \Xi_{2}\right)\right\}
$$

Note that for the sake of simplicity, if $p_{1}=p_{2}=p$, a B-Spline space of degree $p$ should be understood as a B-Spline space of degree $p$ in each parametric direction.

The parametrization of curves and surfaces using B-Spline functions allows an exact representation of a large spectrum of geometries. However, they fail to represent conic sections exactly, which are widely present in the design of various engineering applications. In order to circumvent this, Non-Uniform Rational B-Splines (NURBS) are used instead (see [11] and [34], for instance).

Definition 4 Let $p, k, p_{1}, p_{2}, k_{1}, k_{2} \in \mathbb{N}$ as above. NURBS mappings can be considered as weighted B-Spline mappings. They can be defined as follows;

$$
\begin{gathered}
r(x):=\sum_{i=0}^{k-1} \frac{c_{i} w_{i} b_{i}^{p}(x)}{\sum_{j=0}^{k-1} w_{j} b_{j}^{p}(x)} \quad \text { in 1D, } \\
\mathbf{r}(x):=\sum_{i_{1}=0}^{k_{1}-1} \sum_{i_{2}=0}^{k_{2}-1} \frac{\mathbf{c}_{i_{1}, i_{2}} w_{i_{1}, i_{2}} \cdot b_{i_{1}}^{p_{1}}(x) \cdot b_{i_{2}}^{p_{2}}(x)}{\sum_{j_{1}=0}^{k_{1}-1} \sum_{j_{2}=0}^{k_{2}-1} w_{j_{1}, j_{2}} \cdot b_{j_{1}}^{p_{1}}(x) \cdot b_{j_{2}}^{p_{2}}(x)} \quad \text { in 2D. }
\end{gathered}
$$

Thereby, $w_{i}, w_{i_{1}, i_{2}} \in \mathbb{R}$ are elements of a vector of dimension $k$ and a matrix of dimension $k_{1} \times k_{2}$, containing weighting coefficients of the NURBS, respectively, and $c_{i}, \mathbf{c}_{i_{1}, i_{2}}$ are the control points.

Remark 4 The corresponding univariate NURBS space in the parameter domain is defined analogously to the B-Spline space, namely,

$$
\mathbb{S}_{p}^{w}(\Xi)=\operatorname{span}\left\{\left\{r_{i}^{p}\right\}_{i=0}^{k-1}\right\}
$$

with $r_{i}^{p}(x):=\frac{w_{i} b_{i}^{p}(x)}{\sum_{j=0}^{k-1} w_{j} b_{j}^{p}(x)}$. However, contrary to B-Splines, NURBS spaces on higher dimensional domains cannot be defined using simple tensor product relationships. This is due to the weights $w_{i_{1}, \ldots, i_{d}}$, which cannot be represented in general as a 
tensor product of univariate weights. For convenience, the two-dimensional NURBS space reads

$$
\mathbb{S}_{p_{1}, p_{2}}^{w}\left(\Xi_{1}, \Xi_{2}\right)=\operatorname{span}\left\{\left\{\frac{w_{i_{1}, i_{2}} \cdot b_{i_{1}}^{p_{1}} \cdot b_{i_{2}}^{p_{2}}}{\sum_{j_{1}=0}^{k_{1}-1} \sum_{j_{2}=0}^{k_{2}-1} w_{j_{1}, j_{2}} \cdot b_{j_{1}}^{p_{1}} \cdot b_{j_{2}}^{p_{2}}}\right\}_{i_{1}=0, i_{2}=0}^{k_{1}-1, k_{2}-1}\right\} .
$$

We refer the reader to [4, Section 2], e.g., and the literature cited therein, for a more detailed introduction to NURBS and NURBS spaces.

In order to guarantee the existence of a regular mapping between the parameter and the physical domain, multiple patches defined through a family of regular parameterizations may in some cases be necessary.

Definition 5 Let $\Omega$ be a two-dimensional Lipschitz domain with boundary $\Gamma$. The domain $\Omega$ is called a multipatch domain, if there exists a family of $N_{\Omega}$ disjoint patches such that $\Omega=\bigcup_{i} \Omega_{i}$ and a regular parametrization $\mathbf{r}_{i}(x):[0,1]^{2} \rightarrow$ $\Omega_{i}$ for every single patch $\Omega_{i}$, with $0 \leq i<N_{\Omega}$. Furthermore, we require the parametrization at interfaces to coincide.

Equivalently, $\Gamma$ is also considered a multipatch domain with $\Gamma=\bigcup_{i} \Gamma_{i}$ and $r_{i}(x):[0,1] \rightarrow \Gamma_{i}, \forall \Gamma_{i}$, with $0 \leq i<N_{\Gamma}$.

Knowing that B-Splines form a partition of unity [34], it is easy to see that BSplines are a special type of NURBS, when the weights are equal to 1 .

In the following, if we refer to the geometry, we mean NURBS mappings. If we refer to the spaces used for the discretizations, we mean B-Spline mappings. The motivation for this follows from [7], namely, the spline preserving property of BSplines is needed for a conforming discretization of the De Rham complex.

\subsection{Error estimates for an isogeometric FEM-BEM discretization}

Let the assumptions of Section 2 on $\Omega$ hold. We consider the discrete Problem 3 with $V_{\ell}=\mathbb{S}^{0}(\Omega)$ and $X_{\ell}=\mathbb{S}^{2}(\Gamma)$, where $\mathbb{S}^{0}(\Omega)$ and $\mathbb{S}^{2}(\Gamma)$ are B-Spline spaces defined as in [7] and [44]. Namely,

$$
\mathbb{S}^{0}(\Omega)=\left\{u \in H^{1}(\Omega): u_{\mid \Omega_{i}} \in \mathbb{S}_{p, p}\left(\Omega_{i}\right), \forall 0 \leq i<N_{\Omega}\right\}
$$

and

$$
\mathbb{S}^{2}(\Gamma)=\left\{\phi \in H^{-\frac{1}{2}}(\Gamma): \phi_{\mid \Gamma_{i}} \in \mathbb{S}_{p-1}\left(\Gamma_{i}\right), \forall 0 \leq i<N_{\Gamma}\right\} .
$$

Thereby, $N_{\Omega}$ and $N_{\Gamma}$ denote the number of domain patches and boundary patches, respectively. Note that the degrees of the B-Spline spaces (15) and (16) are solely fixed by one parameter $p>0$.

Definition 6 Let $\Xi=\left\{\xi_{0}, \ldots, \xi_{k+p}\right\}$ be a p-open knot vector. A patch element in the parameter domain is defined as $\left[\xi_{i}, \xi_{i+1}\right]$, for some $0 \leq i<k+p$. The local mesh size is defined as the length of an element, i.e., $h_{i}=\xi_{i+1}-\xi_{i}$. Furthermore, we denote by $h=\max _{0 \leq i<k+p} h_{i}$ the global mesh size of a single patch. Equivalently, $h$ denotes the largest local mesh size of all patches for a multipatch domain. 
Throughout the rest of this work, we assume the following:

(A4) All knot vectors are $p$-open and locally quasi-uniform, i.e., for all non-empty, neighboring elements $\left[\xi_{i_{1}}, \xi_{i_{1}+1}\right]$ and $\left[\xi_{i_{2}}, \xi_{i_{2}+1}\right]$, there exists $\theta \geq 1$, such that

$$
\theta^{-1} \leq h_{i_{1}} h_{i_{2}}^{-1} \leq \theta \text {. }
$$

(A5) The multipatch geometry of $\Omega$ is generated by a family of regular, smooth parameterizations.

Definition 7 Let $D=\{\Omega, \Gamma\}$ be a multipatch domain with $n$ patches. For some $s \in \mathbb{R}$, we define the space of patchwise regularity by

$$
H_{\mathrm{pw}}^{s}(D)=\left\{u \in L^{2}(D):\|u\|_{H_{\mathrm{pw}}^{s}(D)}<\infty\right\},
$$

where

$$
\|u\|_{H_{\mathrm{pw}}^{s}(D)}^{2}=\sum_{0<i \leq n}\left\|u_{\mid D_{i}}\right\|_{H^{s}(D)}^{2}
$$

Lemma 3 Let $u \in H^{1}(\Omega) \cap H_{\mathrm{pw}}^{1+s}(\Omega)$ and $\phi \in H^{-\frac{1}{2}}(\Gamma) \cap H_{\mathrm{pw}}^{-\frac{1}{2}+s}(\Gamma)$. Consider $\mathbb{S}^{0}(\Omega)$ and $\mathbb{S}^{2}(\Gamma)$ as given in (15) and (16), respectively. There exists $C_{0}, C_{2}>0$ depending only on $p$ and $\theta$ such that

$$
\begin{array}{cl}
\inf _{u_{\ell} \in \mathbb{S}^{0}(\Omega)}\left\|u-u_{\ell}\right\|_{H^{1}(\Omega)} \leq C_{0} h^{s}\|u\|_{H_{\mathrm{pw}}^{1+s}(\Omega)}, & 0 \leq s \leq p, \\
\inf _{\phi_{\ell} \in \mathbb{S}^{2}(\Gamma)}\left\|\phi-\phi_{\ell}\right\|_{H^{-\frac{1}{2}}(\Gamma)} \leq C_{2} h^{s}\|\phi\|_{H_{\mathrm{pw}}^{-\frac{1}{2}+s}(\Gamma)}, \quad \frac{1}{2} \leq s \leq p+\frac{1}{2} .
\end{array}
$$

Proof The first estimate is given in [7, Corollary 2], and the second one follows from [7, Corollary 4].

Theorem 4 We assume $C_{\mathrm{ell}}^{\mathcal{U}}>\frac{1}{4}$. Let $(u, \phi) \in \mathcal{H}$ be the solution of the Problem 1 and let $\left(u_{\ell}, \phi_{\ell}\right) \in \mathcal{H}_{\ell}:=\mathbb{S}^{0}(\Omega) \times \mathbb{S}^{2}(\Gamma)$ be the solution of the discrete Problem 3 . Then for $0 \leq s \leq \frac{1}{2}$ and $u \in H^{1}(\Omega) \cap H_{\mathrm{pw}}^{1+s}(\Omega)$ and $\phi \in H^{-\frac{1}{2}}(\Gamma) \cap H_{\mathrm{pw}}^{0}(\Gamma)$

$$
\left\|u-u_{\ell}\right\|_{H^{1}(\Omega)}+\left\|\phi-\phi_{\ell}\right\|_{H^{-\frac{1}{2}}(\Gamma)} \leq C h^{s}\left(\|u\|_{H_{\mathrm{pw}}^{1+s}(\Omega)}+\|\phi\|_{H_{\mathrm{pw}}^{0}(\Gamma)}\right) .
$$

For $\frac{1}{2} \leq s \leq p$, and $u \in H^{1}(\Omega) \cap H_{\mathrm{pw}}^{1+s}(\Omega)$ and $\phi \in H^{-\frac{1}{2}}(\Gamma) \cap H_{\mathrm{pw}}^{-\frac{1}{2}+s}(\Gamma)$, we have

$$
\left\|u-u_{\ell}\right\|_{H^{1}(\Omega)}+\left\|\phi-\phi_{\ell}\right\|_{H^{-\frac{1}{2}(\Gamma)}} \leq C h^{s}\left(\|u\|_{H_{\mathrm{pw}}^{1+s}(\Omega)}+\|\phi\|_{H_{\mathrm{pw}}^{-\frac{1}{2}+s}(\Gamma)}\right)
$$

with a constant $C=C\left(C_{C e ́ a}, p, \theta\right)>0$, which is in particular independent of $h$. 
Proof From [7] we know that $\mathbb{S}^{0}(\Omega)$ and $\mathbb{S}^{2}(\Gamma)$ are closed subspaces of $H^{1}(\Omega)$ and $H^{-\frac{1}{2}}(\Gamma)$, respectively. Moreover, Assumption (A3) is fulfilled per construction of the B-Spline spaces. Hence, the usual analysis for a conforming Galerkin discretization of a non-symmetric FEM-BEM coupling can be considered also in the isogeometric context. Now, using Lemma 3 and the quasi-optimality stated in Theorem 3 yield the assertion.

Remark 5 Throughout this section, we required for the analysis that $C_{\mathrm{ell}}^{\mathcal{U}}>\frac{1}{4}$. However, this assumption is sufficient for the solvability of the Johnson-Nédélec coupling, but not necessary. See also [2] and [15, Remark 10], where numerical experiments still converge although the condition is violated.

\section{Extension of the model problem}

Let $\Omega, \Omega_{1}, \Omega_{\mathrm{b}}, \Omega_{2} \subset \mathbb{R}^{2}$ be bounded Lipschitz domains with diam $\left(\Omega_{\mathrm{b}}\right)<1$ (see Fig. 1). We denote by $\Gamma_{\mathrm{b}}=\Gamma_{1} \cup \Gamma_{2}$ the boundary of $\Omega_{\mathrm{b}}$ and by $\Gamma_{0,1}$ and $\Gamma_{0,2}$ the Dirichlet boundaries of $\Omega_{1}$ and $\Omega_{2}$, respectively. Furthermore, we define

$$
H_{0}^{1}\left(\Omega_{i}, \Gamma_{0, i}\right):=\left\{u \in H^{1}\left(\Omega_{i}\right): u_{\mid \Gamma_{0, i}}=0\right\} \quad \text { for } \quad i=1,2 .
$$

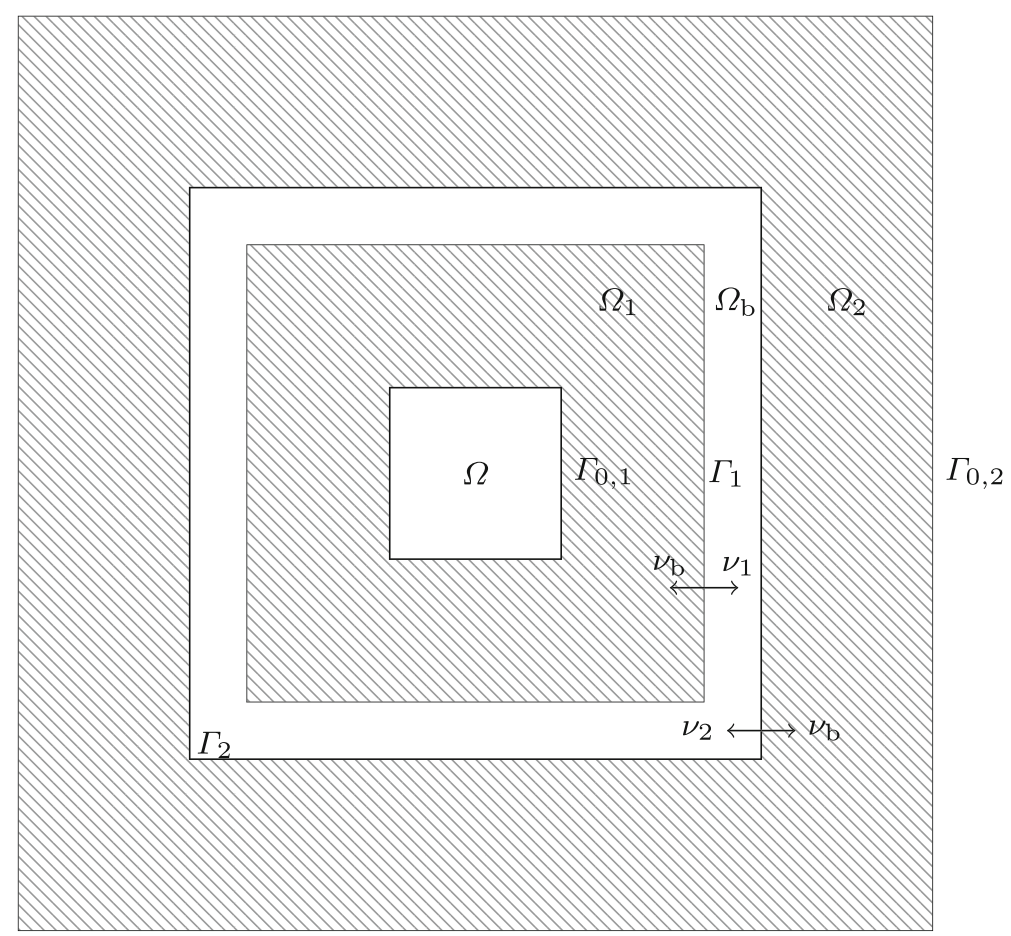

Fig. 1 We see a possible domain arrangement for the boundary value problem discussed in Problem (18) with two disjoint domains separated by a gap. The domain $\Omega_{\mathrm{b}}$ can be very thin and represents, e.g., an air gap. It is surrounded by two bounded domains $\Omega_{1}$ and $\Omega_{2}$ 
We consider the following boundary value problem: Find $\left(u_{1}, u_{2}, u_{\mathrm{b}}\right) \in$ $H_{0}^{1}\left(\Omega_{1}, \Gamma_{0,1}\right) \times H_{0}^{1}\left(\Omega_{2}, \Gamma_{0,2}\right) \times H^{1}\left(\Omega_{\mathrm{b}}\right)$ such that

$$
\begin{aligned}
-\operatorname{div}\left(\mathcal{U}_{i} \nabla u_{i}\right) & =f_{i} & & \text { in } \Omega_{i}, \quad i=1,2, \\
-\Delta u_{\mathrm{b}} & =0 & & \text { in } \Omega_{\mathrm{b}}, \\
u_{\mathrm{b} \mid \Gamma_{i}}-u_{i \mid \Gamma_{i}} & =u_{0, i} & & \text { on } \Gamma_{i}, \quad i=1,2, \\
\mathcal{U}_{i} \nabla u_{i \mid \Gamma_{i}} \cdot v_{i}+\nabla u_{\mathrm{b} \mid \Gamma_{i}} \cdot v_{\mathrm{b}} & =\phi_{0, i} & & \text { on } \Gamma_{i}, \quad i=1,2, \\
u_{i \mid \Gamma_{0, i}} & =0 & & \text { on } \Gamma_{0, i}, \quad i=1,2 .
\end{aligned}
$$

Hereby, $\nu_{i}$ and $\nu_{\mathrm{b}}$ denote the outer normal vector of $\Omega_{i}$ and $\Omega_{\mathrm{b}}$, respectively, $\left(f_{i}, u_{0, i}, \phi_{0, i}\right) \in H_{0}^{1}\left(\Omega_{i}\right)^{\prime} \times H^{\frac{1}{2}}\left(\Gamma_{i}\right) \times H^{-\frac{1}{2}}\left(\Gamma_{i}\right)$ with $i=1,2$ are some given data, and $\mathcal{U}_{i}$ are possibly non-linear operators with the assumptions (A1) and (A2). We emphasize that the model problem (18) can be used to simulate electric machines (see also the example in Section 5.2), which motivates its consideration. Next, we want to derive a weak formulation for model problem (18). We consider the weak form of the two problems in $\Omega_{1}$ and $\Omega_{2}$. Hence, we multiply (18a) with test functions $v_{i}$ and apply the first Green's identity to get

$$
\left(\mathcal{U}_{i} \nabla u_{i}, \nabla v_{i}\right)_{\Omega_{i}}-\left\langle\mathcal{U}_{i} \partial_{\nu_{i}} u_{i}, v_{i \mid \Gamma_{i}}\right\rangle_{\Gamma_{i}}=\left\langle f_{i}, v_{i}\right\rangle_{\Omega_{i}}
$$

for $i=1,2$. Note that $u_{i}=0$ on $\Gamma_{0, i}$. We may transfer (18b) in $\Omega_{\mathrm{b}}$ to an integral equation on $\Gamma_{\mathrm{b}}$ in order to apply BEM in the following. Hence, the (interior) representation formula (4) $(\kappa=0)$ hold if we replace $u$ by $u_{\mathrm{b}}$. Let $\phi:=\partial_{\nu_{\mathrm{b}}} u_{\mathrm{b}}$ denote the conormal derivative of $u_{\mathrm{b}}$ on $\Gamma_{\mathrm{b}}$, the BIE is obtained as in Section 1

$$
\mathcal{V} \phi=\left(\frac{1}{2}+\mathcal{K}\right) u_{\left.\mathrm{b}\right|_{\Gamma_{\mathrm{b}}}},
$$

where the single layer operator $\mathcal{V}$ and the double layer operator $\mathcal{K}$ are defined in (6) over $\Gamma_{\mathrm{b}}$ instead of $\Gamma$ but of course with the same fundamental solution $G(x, y)$. Note that the normal vector $\nu_{\mathrm{b}}$ points outwards with respect to $\Omega_{\mathrm{b}}$ since it is considered as an interior problem in our integral equation notation.

In what follows we strongly follow the work of [32], where a boundary value problem with hard inclusion is considered. As in [32], we can derive two equivalent weak formulations. It is enough to consider here only one. The following considerations might help for a better understanding for the weak coupling formulation below. Note that for a constant it follows $\left(\frac{1}{2}+\mathcal{K}\right) 1=0$ on $\Gamma_{\mathrm{b}}$. Furthermore, if $\mathcal{K}^{\prime}$ is the adjoint operator of $\mathcal{K}$ and we have $\mathcal{V}^{-1} \mathcal{K}=\mathcal{K}^{\prime} \mathcal{V}^{-1}$. Then, with (20) we see that

$$
\begin{aligned}
\langle\phi, 1\rangle_{\Gamma_{\mathrm{b}}} & =\left\langle\mathcal{V} \phi, \mathcal{V}^{-1} 1\right\rangle_{\Gamma_{\mathrm{b}}}=\left\langle\left(\frac{1}{2}+\mathcal{K}\right) u_{\mathrm{b} \mid \Gamma_{\mathrm{b}}}, \mathcal{V}^{-1} 1\right\rangle_{\Gamma_{\mathrm{b}}}=\left\langle u_{\mathrm{b} \mid \Gamma_{\mathrm{b}}},\left(\frac{1}{2}+\mathcal{K}^{\prime}\right) \mathcal{V}^{-1} 1\right\rangle_{\Gamma_{\mathrm{b}}} \\
& =\left\langle u_{\mathrm{b} \mid \Gamma_{\mathrm{b}}}, \mathcal{V}^{-1}\left(\frac{1}{2}+\mathcal{K}\right) 1\right\rangle_{\Gamma_{\mathrm{b}}}=0 .
\end{aligned}
$$

Note that this $\phi$ together with the representation formula leads to $u_{\mathrm{b}}$ in $\Omega_{\mathrm{b}}$ (see also [30, Theorem 7.5]). Therefore, we introduce the following subspace

$$
H_{\star}^{-\frac{1}{2}}\left(\Gamma_{\mathrm{b}}\right)=\left\{\psi \in H^{-\frac{1}{2}}\left(\Gamma_{\mathrm{b}}\right):\left\langle\psi, 1_{\Gamma_{\mathrm{b}}}\right\rangle_{\Gamma_{\mathrm{b}}}=0\right\} .
$$


Furthermore, similar as in Section 2 we introduce a product space with its norm, namely

$$
\begin{aligned}
\mathcal{H}_{0} & :=H_{0}^{1}\left(\Omega_{1}, \Gamma_{0,1}\right) \times H_{0}^{1}\left(\Omega_{2}, \Gamma_{0,2}\right) \times H_{\star}^{-\frac{1}{2}}\left(\Gamma_{\mathrm{b}}\right), \\
\|\mathbf{v}\|_{\mathcal{H}_{0}} & :=\left(\left\|v_{1}\right\|_{H^{1}\left(\Omega_{1}\right)}^{2}+\left\|v_{2}\right\|_{H^{1}\left(\Omega_{2}\right)}^{2}+\|\psi\|_{H^{-\frac{1}{2}\left(\Gamma_{\mathrm{b}}\right)}}^{2}\right)^{\frac{1}{2}} \text { for } \mathbf{v}:=\left(v_{1}, v_{2}, \psi\right) \in \mathcal{H}_{0} .
\end{aligned}
$$

Remark 6 Instead of considering a subspace and thus eliminating the constants from the solution space, a suitable orthogonal decomposition of $H^{-\frac{1}{2}}\left(\Gamma_{\mathrm{b}}\right)$ in the following proofs could also be considered (see [32]).

Using $\Gamma_{\mathrm{b}}=\Gamma_{1} \cup \Gamma_{2}$ and inserting the corresponding jump conditions (18c) and (18d) in (20) and (19), respectively, yield the following weak formulation for problem (18):

Find $\mathbf{u}:=\left(u_{1}, u_{2}, \phi\right) \in \mathcal{H}_{0}:=H_{0}^{1}\left(\Omega_{1}, \Gamma_{0,1}\right) \times H_{0}^{1}\left(\Omega_{2}, \Gamma_{0,2}\right) \times H_{\star}^{-\frac{1}{2}}\left(\Gamma_{\mathrm{b}}\right)$ such that

$$
\begin{aligned}
\left(\mathcal{U}_{1} \nabla u_{1}, \nabla v_{1}\right)_{\Omega_{1}}+\left\langle\phi_{\mid \Gamma_{1}}, v_{1 \mid \Gamma_{1}}\right\rangle_{\Gamma_{1}} & =\left\langle f_{1}, v_{1}\right\rangle_{\Omega_{1}}+\left\langle\phi_{0,1}, v_{1 \mid \Gamma_{1}}\right\rangle_{\Gamma_{1}}, \\
\left(\mathcal{U}_{2} \nabla u_{2}, \nabla v_{2}\right)_{\Omega_{2}}+\left\langle\phi_{\mid \Gamma_{2}}, v_{2 \mid \Gamma_{2}}\right\rangle_{\Gamma_{2}} & =\left\langle f_{2}, v_{2}\right\rangle_{\Omega_{2}}+\left\langle\phi_{0,2}, v_{2 \mid \Gamma_{2}}\right\rangle_{\Gamma_{2}}, \\
\langle\psi, \mathcal{V} \phi\rangle_{\Gamma_{\mathrm{b}}}-\sum_{i=1}^{2}\left\langle\psi,\left(\frac{1}{2}+\mathcal{K}\right) u_{i \mid \Gamma_{i}}\right\rangle_{\Gamma_{\mathrm{b}}} & =\sum_{i=1}^{2}\left\langle\psi,\left(\frac{1}{2}+\mathcal{K}\right) u_{0, i}\right\rangle_{\Gamma_{\mathrm{b}}}
\end{aligned}
$$

hold $\forall \mathbf{v}:=\left(v_{1}, v_{2}, \psi\right) \in \mathcal{H}_{0}$.

As before we first write the problem in a compact form.

Problem 4 Find $\mathbf{u}:=\left(u_{1}, u_{2}, \phi\right) \in \mathcal{H}_{0}$ such that $b(\mathbf{u}, \mathbf{v})=\iota(\mathbf{v})$ holds $\forall \mathbf{v}:=$ $\left(v_{1}, v_{2}, \psi\right) \in \mathcal{H}_{0}$.

Thereby,

$$
\begin{aligned}
b(\mathbf{u}, \mathbf{v}):= & \sum_{i=1}^{2}\left(\left(\mathcal{U}_{i} \nabla u_{i}, \nabla v_{i}\right)_{\Omega_{i}}+\left\langle\phi_{\mid \Gamma_{i}}, v_{i \mid \Gamma_{i}}\right\rangle_{\Gamma_{i}}-\left\langle\psi,\left(\frac{1}{2}+\mathcal{K}\right) u_{i \mid \Gamma_{i}}\right\rangle_{\Gamma_{\mathrm{b}}}\right) \\
& +\langle\psi, \mathcal{V} \phi\rangle_{\Gamma_{\mathrm{b}}}
\end{aligned}
$$

and

$$
\iota(\mathbf{v}):=\sum_{i=1}^{2}\left(\left\langle f_{i}, v_{i}\right\rangle_{\Omega_{i}}+\left\langle\phi_{0, i}, v_{i \mid \Gamma_{i}}\right\rangle_{\Gamma_{i}}+\left\langle\psi,\left(\frac{1}{2}+\mathcal{K}\right) u_{0, i}\right\rangle_{\Gamma_{\mathrm{b}}}\right) .
$$

In this case no stabilization is needed, since both subproblems involve a Dirichlet boundary condition. Hence, we prove directly the strong monotonicity of $b(\cdot, \cdot)$. Equivalently to (13), the form $b(\cdot, \cdot)$ induces a non-linear operator $\mathcal{B}: \mathcal{H}_{0} \rightarrow \mathcal{H}_{0}^{\prime}$ with

$$
\langle\mathcal{B}(\mathbf{u}), \mathbf{v}\rangle:=b(\mathbf{u}, \mathbf{v}) \quad \forall \mathbf{u}, \mathbf{v} \in \mathcal{H}_{0} .
$$

The next theorem states the strong monotonicity of the method for the extended BVP. It can be considered as an extension to our problem setting of the stability estimate 
result given in [32] for an interior Dirichlet BVP of a diffusion equation with a hard inclusion. The key idea therein is to estimate the energy of the bounded finite element domains with the energy of some related problem in the exterior domain. Let $\lambda>0$ be the minimal eigenvalue of the related exterior problem. If both corresponding Steklov-Poincaré operators are $H^{\frac{1}{2}}(\Gamma)$-elliptic, then we have that

$$
\lambda\left\langle S^{\mathrm{ext}} v, v\right\rangle \leq\left\langle S^{\mathrm{int}} v, v\right\rangle, \text { for all } v \in H^{\frac{1}{2}}(\Gamma),
$$

where $S^{\text {ext }}$ and $S^{\text {int }}$ are the Steklov-Poincare operators of the exterior and the interior domain, respectively, c.f. [32]. The constants in the following theorem have similar dependencies as described in Remark 2.

Theorem 5 Let us consider the non-linear operator $\mathcal{B}: \mathcal{H}_{0} \rightarrow \mathcal{H}_{0}^{\prime}$ defined in (22) with $\mathcal{H}_{0}:=H_{0}^{1}\left(\Omega_{1}, \Gamma_{0,1}\right) \times H_{0}^{1}\left(\Omega_{2}, \Gamma_{0,2}\right) \times H_{\star}^{-\frac{1}{2}}\left(\Gamma_{\mathrm{b}}\right)$. Furthermore, $\lambda_{1}, \lambda_{2}>0$ are the eigenvalues of (23) with respect to the domains $\Omega_{1}$ and $\Omega_{2}$. We state the following three assertions:

1. $\mathcal{B}$ is Lipschitz continuous, i.e., there exists $C_{\mathrm{Lip}}>0$ such that

$$
\|\mathcal{B}(\mathbf{u})-\mathcal{B}(\mathbf{v})\|_{\mathcal{H}_{0}^{\prime}} \leq C_{\text {Lip }}\|\mathbf{u}-\mathbf{v}\|_{\mathcal{H}_{0}}
$$

for all $\mathbf{u}, \mathbf{v} \in \mathcal{H}_{0}$.

2. if $C_{\mathrm{ell}}^{\mathcal{U}_{i}}>\frac{1}{4 \lambda_{i}}$ for $i=1,2$ then

$$
\begin{aligned}
\langle\mathcal{B}(\mathbf{u})-\mathcal{B}(\mathbf{v}), \mathbf{u}-\mathbf{v}\rangle \geq & C_{\text {stab }}\left(\left\|\nabla u_{1}-\nabla v_{1}\right\|_{L^{2}\left(\Omega_{1}\right)}^{2}+\left\|\nabla u_{2}-\nabla v_{2}\right\|_{L^{2}\left(\Omega_{2}\right)}^{2}\right. \\
& \left.+\|\phi-\psi\|_{\mathcal{V}}^{2}\right)
\end{aligned}
$$

for all $\mathbf{u}:=\left(u_{1}, u_{2}, \phi\right) \in \mathcal{H}_{0}, \mathbf{v}:=\left(v_{1}, v_{2}, \psi\right) \in \mathcal{H}_{0}$ with

$$
C_{\text {stab }}=\min \left\{1, \frac{1}{2}\left(1+C_{\mathrm{ell}}^{\mathcal{U}_{1}}-\sqrt{\left(C_{\mathrm{ell}}^{\mathcal{U}_{1}}-1\right)^{2}+\frac{1}{\lambda_{1}}}\right), \frac{1}{2}\left(1+C_{\mathrm{ell}}^{\mathcal{U}_{2}}-\sqrt{\left(C_{\mathrm{ell}}^{\mathcal{U}_{2}}-1\right)^{2}+\frac{1}{\lambda_{2}}}\right)\right\} .
$$

3. if $C_{\mathrm{ell}}^{\mathcal{U}_{i}}>\frac{1}{4 \lambda_{i}}$ for $i=1,2$, then $\mathcal{B}$ is strongly monotone, i.e., there exists $C_{\mathrm{ell}}>0$ such that

$$
\langle\mathcal{B}(\mathbf{u})-\mathcal{B}(\mathbf{v}), \mathbf{u}-\mathbf{v}\rangle \geq C_{\text {ell }}\|\mathbf{u}-\mathbf{v}\|_{\mathcal{H}_{0}}^{2}
$$

for all $\mathbf{u}, \mathbf{v} \in \mathcal{H}_{0}$.

Proof The Lipschitz continuity follows merely from the Lipschitz continuity of $\mathcal{U}_{1}$ and $\mathcal{U}_{2}$ and the continuity of the boundary integral operators.

The stability estimate follows strongly the steps of the proofs of [32, Theorem 2.2.ii.] and in [32, Section 5.1]. Since we are dealing with a different BVP and non-linear material tensors, we sketch the main steps of the proof, for convenience. 
For ease of notation, let $\mathbf{w}:=\left(w_{1}, w_{2}, \xi\right)=\mathbf{u}-\mathbf{v}=\left(u_{1}-v_{1}, u_{2}-v_{2}, \phi-\psi\right) \in$ $\mathcal{H}_{0}$. From (22), we get

$$
\begin{aligned}
\langle\mathcal{B}(\mathbf{u})-\mathcal{B}(\mathbf{v}), \mathbf{w}\rangle:= & \sum_{i=1}^{2}\left(\left(\mathcal{U}_{i} \nabla u_{i}-\mathcal{U}_{i} \nabla v_{i}, \nabla w_{i}\right)_{\Omega_{i}}+\left\langle\xi,\left.\left(\frac{1}{2}-\mathcal{K}\right) w_{i \mid \Gamma_{i}}\right|_{\Gamma_{\mathrm{b}}}\right)\right. \\
& +\langle\xi, \mathcal{V} \xi\rangle_{\Gamma_{\mathrm{b}}} .
\end{aligned}
$$

First, we start with the domain parts. Provided $\mathcal{U}_{i}, i=1,2$, are strongly monotone, then it holds

$$
\left(\mathcal{U}_{i} \nabla u_{i}-\mathcal{U}_{i} \nabla v_{i}, \nabla w_{i}\right)_{\Omega_{i}} \geq C_{\mathrm{ell}}^{\mathcal{U}_{i}}\left\|\nabla w_{i}\right\|_{L^{2}\left(\Omega_{i}\right)}^{2} .
$$

For $w_{i} \in H_{0}^{1}\left(\Omega_{i}, \Gamma_{0, i}\right)$, we now consider the splitting $w_{i}=\bar{w}_{i}+w_{0, i}$, where $\bar{w}_{i}$ is the harmonic extension of $w_{i \mid \Gamma_{i}}$ and $w_{0, i} \in H_{0}^{1}\left(\Omega_{i}, \partial \Omega_{i}\right)$ (see, e.g., [15]). From this follows

$$
\left\|\nabla w_{i}\right\|_{L^{2}\left(\Omega_{i}\right)}^{2}=\left\|\nabla w_{0, i}\right\|_{L^{2}\left(\Omega_{i}\right)}^{2}+\left\langle S_{i} w_{i \mid \Gamma_{i}}, w_{i \mid \Gamma_{i}}\right\rangle_{\Gamma_{i}},
$$

where $S_{i}, i=1,2$, denote the interior Steklov-Poincaré operators of the bounded domains $\Omega_{1}$ and $\Omega_{2}$, respectively. Hence,

$$
\left(\mathcal{U}_{i} \nabla u_{i}-\mathcal{U}_{i} \nabla v_{i}, \nabla w_{i}\right)_{\Omega_{i}} \geq C_{\text {ell }}^{\mathcal{U}_{i}}\left(\left\|\nabla w_{0, i}\right\|_{L^{2}\left(\Omega_{i}\right)}^{2}+\left\langle S_{i} w_{i \mid \Gamma_{i}}, w_{i \mid \Gamma_{i}}\right\rangle_{\Gamma_{i}}\right)
$$

Next, by using the contractivity of $\mathcal{K}$, as given in [32, Lemma 2.1] (we consider here the worst case $C_{\mathcal{K}}=1$ ), as well as the invertibility of $\mathcal{V}$, we obtain

$$
\left\langle\xi,\left(\frac{1}{2}-\mathcal{K}\right) w_{i \mid \Gamma_{i}}\right\rangle_{\Gamma_{\mathrm{b}}} \leq\|\xi\|_{\mathcal{V}} \sqrt{\left\langle S_{i}^{\mathrm{ext}} w_{i \mid \Gamma_{i}}, w_{i \mid \Gamma_{i}}\right\rangle_{\Gamma_{\mathrm{b}}}}, \quad i=1,2,
$$

where $S_{i}^{\text {ext }}: H^{\frac{1}{2}}\left(\Gamma_{\mathrm{b}}\right) \rightarrow H^{-\frac{1}{2}}\left(\Gamma_{\mathrm{b}}\right)$ are the Steklov-Poincaré operators associated to the corresponding exterior eigenvalue problem (see [32, Section 2.2]). Similarly, we assume the following spectral equivalence

$$
\left\langle S_{i}^{\text {ext }} w_{i \mid \Gamma_{i}}, w_{i \mid \Gamma_{i}}\right\rangle_{\Gamma_{i}} \leq \frac{1}{\lambda_{i}}\left\langle S_{i} w_{i \mid \Gamma_{i}}, w_{i \mid \Gamma_{i}}\right\rangle_{\Gamma_{i}} \text {, for all } w_{i} \in H_{0}^{1}\left(\Omega_{i}, \Gamma_{0, i}\right),
$$

where $\lambda_{i}, i=1,2$ are characterized as minimal eigenvalues of the related problem. Thus,

$$
\left\langle\xi,\left(\frac{1}{2}-\mathcal{K}\right) w_{i \mid \Gamma_{i}}\right\rangle_{\Gamma_{\mathrm{b}}} \leq\|\xi\|_{\mathcal{V}} \sqrt{\frac{1}{\lambda_{i}}\left\langle S_{i} w_{i \mid \Gamma_{i}}, w_{i \mid \Gamma_{i}}\right\rangle_{\Gamma_{i}}}, \quad i=1,2
$$

Inserting (28) and (29) in (27), $\langle\xi, \mathcal{V} \xi\rangle_{\Gamma_{\mathrm{b}}}=\|\xi\|_{\mathcal{V}}^{2}$ and some manipulations as in the proof of [15, Theorem1] lead to the assertion.

To prove the last claim we consider $\mathbf{v}:=\left(v_{1}, v_{2}, \psi\right) \in \mathcal{H}_{0}$. Note that $v_{1}=0$ on $\Gamma_{0,1}$ and $v_{2}=0$ on $\Gamma_{0,2}$ with $\left|\Gamma_{0,1}\right|,\left|\Gamma_{0,2}\right|>0$. Due to Friedrichs's inequality and (7) it follows that $\left\|\nabla v_{1}\right\|_{L^{2}\left(\Omega_{1}\right)}^{2}+\left\|\nabla v_{2}\right\|_{L^{2}\left(\Omega_{2}\right)}^{2}+\|\psi\|_{\mathcal{V}}^{2}$ is an equivalent norm on $\mathcal{H}_{0}$. Thus, (26) follows directly from (25).

Equivalently to Theorem 2, the strong monotonicity and the Lipschitz continuity of the non-linear operator $\mathcal{B}$ provide the well-posedness of Problem 4 for any $\left(f_{i}, u_{0, i}, \phi_{0, i}\right) \in H_{0}^{1}\left(\Omega_{i}\right)^{\prime} \times H^{\frac{1}{2}}\left(\Gamma_{i}\right) \times H^{-\frac{1}{2}}\left(\Gamma_{i}\right)$ with $i=1,2$. 
As for the interface problem, we consider a conforming Galerkin discretization in the sense of an isogeometric FEM-BEM discretization. Namely, the discrete problem is obtained by replacing $\mathbf{u}:=\left(u_{1}, u_{2}, \phi\right) \in \mathcal{H}_{0}:=H_{0}^{1}\left(\Omega_{1}, \Gamma_{0,1}\right) \times H_{0}^{1}\left(\Omega_{2}, \Gamma_{0,2}\right) \times$ $H_{\star}^{-\frac{1}{2}}\left(\Gamma_{\mathrm{b}}\right)$ in Problem 4 with $\mathbf{u}_{\ell}:=\left(u_{1, \ell}, u_{2, \ell}, \phi_{\ell}\right) \in \mathcal{H}_{0, \ell}:=\mathbb{S}^{0}\left(\Omega_{1}, \Gamma_{0,1}\right) \times$ $\mathbb{S}^{0}\left(\Omega_{2}, \Gamma_{0,2}\right) \times \mathbb{S}^{2}\left(\Gamma_{\mathrm{b}}\right)$. Note that accordingly to the notation in the continuous setting, $\mathbb{S}^{0}(\Omega, \Gamma)$ denotes the B-Spline space $\mathbb{S}^{0}(\Omega)$ of order $p$ as defined in (15) with a Dirichlet boundary $\Gamma \subseteq \partial \Omega$ and $\mathbb{S}^{2}\left(\Gamma_{\mathrm{b}}\right)$ is defined in (16).

Problem 5 Find $\mathbf{u}_{\ell}:=\left(u_{1, \ell}, u_{2, \ell}, \phi_{\ell}\right) \in \mathcal{H}_{0, \ell}:=\mathbb{S}^{0}\left(\Omega_{1}, \Gamma_{0,1}\right) \times \mathbb{S}^{0}\left(\Omega_{2}, \Gamma_{0,2}\right) \times$ $\mathbb{S}^{2}\left(\Gamma_{\mathrm{b}}\right)$ such that $b\left(\mathbf{u}_{\ell}, \mathbf{v}_{\ell}\right)=\iota\left(\mathbf{v}_{\ell}\right)$ holds $\forall \mathbf{v}_{\ell}:=\left(v_{1, \ell}, v_{2, \ell}, \psi_{\ell}\right) \in \mathcal{H}_{0, \ell}$.

Analogously to the interface problem, we state in the following theorem the quasioptimality in the sense of the Céa-type Lemma of the Galerkin discretization of Problem 4, as well as an a priori error estimate for the introduced B-Spline discretization. To simplify the presentation, we introduce in this section a piecewise defined product space

$$
\begin{aligned}
\mathcal{H}_{\mathrm{pw}}^{s}:= & \left(H^{1}\left(\Omega_{1}\right) \cap H_{\mathrm{pw}}^{1+s}\left(\Omega_{1}\right)\right) \times\left(H^{1}\left(\Omega_{2}\right) \cap H_{\mathrm{pw}}^{1+s}\left(\Omega_{2}\right)\right) \\
& \times\left(H_{\star}^{-\frac{1}{2}}\left(\Gamma_{\mathrm{b}}\right) \cap H_{\mathrm{pw}}^{-\frac{1}{2}+s}\left(\Gamma_{\mathrm{b}}\right)\right)
\end{aligned}
$$

for $s \geq 0$, which is used to get convergence rates with the aid of Lemma 3. The corresponding norm defined in the sense of (17) is denoted by $\|\cdot\|_{\mathcal{H}_{\mathrm{pw}}^{s}}$.

Theorem 6 For $i=1,2$, let $C_{\mathrm{ell}}^{\mathcal{U}_{i}}>\frac{1}{4 \lambda_{i}}$, where $\lambda_{i}>0$ are the eigenvalues of (23) with respect to the domains $\Omega_{i}$. Moreover, let $\mathbf{u} \in \mathcal{H}_{0}$ be the solution of Problem 4 and $\mathbf{u}_{\ell} \in \mathcal{H}_{0, \ell}$ be the discrete solution of Problem 5. Then we have the following results:

- Quasi-optimality:

$$
\left\|\mathbf{u}-\mathbf{u}_{\ell}\right\|_{\mathcal{H}_{0}} \leq C_{C e ́ a} \min _{\mathbf{v}_{\ell} \in \mathcal{H}_{0, \ell}}\left\|\mathbf{u}-\mathbf{v}_{\ell}\right\|_{\mathcal{H}_{0}},
$$

where $C_{\text {Céa }}=\frac{C_{\mathrm{Lip}}}{C_{\mathrm{ell}}}$.

- A priori estimate: For $\frac{1}{2} \leq s \leq p$ and $\mathbf{u} \in \mathcal{H}_{\mathrm{pw}}^{s}$

$$
\left\|\mathbf{u}-\mathbf{u}_{\ell}\right\|_{\mathcal{H}_{0}} \leq C h^{s}\|\mathbf{u}\|_{\mathcal{H}_{\mathrm{pw}}^{s}}
$$

with a constant $C=C\left(C_{C e ́ a}, p, \theta\right)>0$, which is independent of $h$. For $0 \leq s \leq$ $\frac{1}{2}$, we get a similar result as stated in Theorem 4 with $\phi \in H_{\star}^{-\frac{1}{2}}\left(\Gamma_{\mathrm{b}}\right) \cap H_{\mathrm{pw}}^{0}\left(\Gamma_{\mathrm{b}}\right)$.

Proof Quasi-optimality follows from the strong monotonicity and Lipschitz continuity stated in Theorem 5, by following the lines of Theorem 3. The a priori estimate follows from the quasi-optimality and Lemma 3, as is done in Theorem 4 for the interface problem. 
The non-linear operators $\mathcal{U}_{i}, i=1,2$, are now considered to have the form $\mathcal{U}_{i} \nabla u:=g_{i}(|\nabla u|) \nabla u$ with non-linear functions $g_{i}: \mathbb{R} \rightarrow \mathbb{R}$. Similarly to the interface problem, we state the following stability result.

Lemma 4 Let $C_{\mathrm{ell}}^{\mathcal{U}_{i}}>\frac{1}{4 \lambda_{i}}, i=1,2$, with $\lambda_{i}$ as in Theorem 6. Furthermore, the nonlinear operators $\mathcal{U}_{i}, i=1,2$, shall have the form $\mathcal{U}_{i} \nabla u:=g_{i}(|\nabla u|) \nabla u$ with the non-linear functions $g_{i}: \mathbb{R} \rightarrow \mathbb{R}$. Moreover, let $\mathbf{u} \in \mathcal{H}_{0}$ be the unique solution of Problem 4 and $\left(f_{i}, u_{0, i}, \phi_{0, i}\right) \in H_{0}^{1}\left(\Omega_{i}\right)^{\prime} \times H^{\frac{1}{2}}\left(\Gamma_{i}\right) \times H^{-\frac{1}{2}}\left(\Gamma_{i}\right)$, with $i=1,2$, be some suitable inputs. There exists $C>0$ such that

$$
\|\mathbf{u}\|_{\mathcal{H}_{0}} \leq C \sum_{i=1}^{2}\left(\left\|f_{i}\right\|_{H^{1}\left(\Omega_{i}\right)^{\prime}}+\left\|u_{i, 0}\right\|_{H^{\frac{1}{2}\left(\Gamma_{i}\right)}}+\left\|\phi_{i, 0}\right\|_{H^{-\frac{1}{2}\left(\Gamma_{i}\right)}}\right) .
$$

Proof We know from the strong monotonicity of $\mathcal{B}$ that

$$
C_{\text {ell }}\|\mathbf{u}-\mathbf{v}\|_{\mathcal{H}_{0}}^{2} \leq\langle\mathcal{B}(\mathbf{u})-\mathcal{B}(\mathbf{v}), \mathbf{u}-\mathbf{v}\rangle
$$

holds for all $\mathbf{u}, \mathbf{v} \in \mathcal{H}_{0}$. Without loss of generality, we choose $\mathbf{v}=(0,0,0)$ and note that $\mathcal{U}_{i} \nabla v_{i}=0, i=1,2$, for our specific non-linearity. Since $\mathbf{u}:=\left(u_{1}, u_{2}, \phi\right)$ is the unique solution of the problem, we conclude that

$$
\begin{aligned}
C_{\mathrm{ell}}\|\mathbf{u}\|_{\mathcal{H}_{0}}^{2} & \leq\langle\mathcal{B}(\mathbf{u}), \mathbf{u}\rangle=\iota(\mathbf{u}), \\
& =\sum_{i=1}^{2}\left(\left\langle f_{i}, u_{i}\right\rangle_{\Omega_{i}}+\left\langle\phi_{0, i}, u_{i \mid \Gamma_{i}}\right\rangle_{\Gamma_{i}}+\left\langle\phi,\left(\frac{1}{2}+\mathcal{K}\right) u_{0, i}\right\rangle_{\Gamma_{\mathrm{b}}}\right) .
\end{aligned}
$$

Using inequality (8) along with the boundedness of $\mathcal{K}$, and rearranging the terms provides the assertion.

In many practical applications, one is not directly interested in the solution $\left(u_{1}, u_{2}\right.$, $\phi)$ of Problem 4 rather than in some derived quantities. These quantities are, for example, evaluated in the exterior/air gap domain. As it can be observed for standalone BEM applications, estimating the error in functionals of the solution may lead to so called super-convergence, i.e., linear functionals of the solution may converge better than the solution in the energy norm (see [37, Section 4.2.5]). With enough regularity the convergence rate doubles.

In the following, this behavior is also shown for the coupled problem. For this, we use the following Aubin-Nitsche argument, similar to [37, Theorem 4.2.14].

Theorem 7 Let $\mathfrak{F} \in \mathcal{H}_{0}^{\prime}$ be a continuous and linear functional, $\mathbf{u}:=\left(u_{1}, u_{2}, \phi\right) \in$ $\mathcal{H}_{0}:=H_{0}^{1}\left(\Omega_{1}, \Gamma_{0,1}\right) \times H_{0}^{1}\left(\Omega_{2}, \Gamma_{0,2}\right) \times H_{\star}^{-\frac{1}{2}}\left(\Gamma_{\mathrm{b}}\right)$ be the solution of Problem 4 and $\mathbf{u}_{\ell}:=\left(u_{1, \ell}, u_{2, \ell}, \phi_{\ell}\right) \in \mathcal{H}_{0, \ell}$ be the discrete solution of Problem 5. Furthermore, $\mathbf{w} \in \mathcal{H}_{0}$ is the unique solution of the dual problem

$$
b(\mathbf{v}, \mathbf{w})=\mathfrak{F}(\mathbf{v})
$$


for all $\mathbf{v} \in \mathcal{H}_{0}$. Then there exists a constant $C_{1}=C_{1}\left(C_{\mathrm{Lip}}\right)>0$ such that

$$
\left|\mathfrak{F}(\mathbf{u})-\mathfrak{F}\left(\mathbf{u}_{\ell}\right)\right| \leq C_{1}\left\|\mathbf{u}-\mathbf{v}_{\ell}\right\|_{\mathcal{H}_{0}}\left\|\mathbf{w}-\mathbf{z}_{\ell}\right\|_{\mathcal{H}_{0}}
$$

for arbitrary $\mathbf{v}_{\ell} \in \mathcal{H}_{0, \ell}, \mathbf{z}_{\ell} \in \mathcal{H}_{0, \ell}$. Furthermore, let $\frac{1}{2} \leq s, t \leq p$ and remember the product space defined in (30). Provided $\mathbf{u}$ and $\mathbf{w}$ are additionally in $\mathcal{H}_{\mathrm{pw}}^{s}$ and $\mathcal{H}_{\mathrm{pw}}^{t}$, respectively, there exists a constant $C_{2}=C_{2}\left(C_{\mathrm{Lip}}, p, \theta\right)>0$ such that

$$
\left|\mathfrak{F}(\mathbf{u})-\mathfrak{F}\left(\mathbf{u}_{\ell}\right)\right| \leq C_{2} h^{s+t}\|\mathbf{u}\|_{\mathcal{H}_{\mathrm{pw}}^{s}}\|\mathbf{w}\|_{\mathcal{H}_{\mathrm{pw}}^{t}} .
$$

Proof The proof follows strongly the lines in [37, Theorem 4.2.14]. Since we allow non-linearities, we give a brief sketch. First of all, we note that Theorem 5 holds for arbitrary functions. Thus, well-posedness, and hence the existence of a unique solution can be established also for the dual problem (32). Furthermore, the dual problem (32), the Galerkin orthogonality $b\left(\mathbf{u}-\mathbf{u}_{\ell}, \mathbf{z}_{\ell}\right)=0$ for all $\mathbf{z}_{\ell} \in \mathcal{H}_{0, \ell}$, and the Lipschitz continuity of the form $b(\cdot, \cdot)$ provide

$$
\left|\mathfrak{F}(\mathbf{u})-\mathfrak{F}\left(\mathbf{u}_{\ell}\right)\right|=\left|\mathfrak{F}\left(\mathbf{u}-\mathbf{u}_{\ell}\right)\right|=\left|b\left(\mathbf{u}-\mathbf{u}_{\ell}, \mathbf{w}-\mathbf{z}_{\ell}\right)\right| \leq C_{1}\left\|\mathbf{u}-\mathbf{u}_{\ell}\right\|_{\mathcal{H}_{0}}\left\|\mathbf{w}-\mathbf{z}_{\ell}\right\|_{\mathcal{H}_{0}}
$$

for arbitrary $\mathbf{z}_{\ell}:=\left(z_{1, \ell}, z_{2, \ell}, \varphi_{\ell}\right) \in \mathcal{H}_{0, \ell}$.

With (31) we get the claim (33). Since Lemma 3 holds for arbitrary $\mathbf{u}$, (34) follows from (33).

Remark 7 In practice the functional of Theorem 7 may be, e.g., the representation formula of the BEM part $\Omega_{\mathrm{b}}$, i.e., for $\mathbf{u}:=\left(u_{1}, u_{2}, \phi\right) \in \mathcal{H}_{0}$

$$
u_{\mathrm{b}}(x)=\mathfrak{F}(\mathbf{u}):=\sum_{i=1}^{2}\left(\int_{\Gamma_{i}} G(x, y) \phi_{\mid \Gamma_{i}} \mathrm{~d} \sigma_{y}-\int_{\Gamma_{i}} \partial_{\nu(y)} G(x, y)\left(u_{i \mid \Gamma_{i}}+u_{0, i \mid \Gamma_{i}}\right) \mathrm{d} \sigma_{y}\right) .
$$

Next, let us assume the regularity $\mathbf{u} \in \mathcal{H}_{\mathrm{pw}}^{p}$ for the solution of Problem 4 and $\mathbf{w} \in$ $\mathcal{H}_{\mathrm{pw}}^{p}$ of its dual problem (32), where the spaces are defined in (30). Then with the discrete solution $\mathbf{u}_{\ell} \in \mathcal{H}_{0, \ell}$ of Problem 5 and (34) we calculate the pointwise error in $\Omega_{\mathrm{b}}$ as

$$
\left|u_{\mathrm{b}}(x)-u_{\mathrm{b}, \ell}(x)\right|=\left|\mathfrak{F}(\mathbf{u})(x)-\mathfrak{F}\left(\mathbf{u}_{\ell}\right)(x)\right| \leq C h^{2 p},
$$

which is the maximal possible super-convergence. Since the constant $C$ depends on $\|\mathbf{u}\|_{\mathcal{H}_{\mathrm{pw}}^{p}}$ and $\|\mathbf{w}\|_{\mathcal{H}_{\mathrm{pw}}^{p}}$, a possible estimate of these norms would probably involve their right-hand sides. The right-hand side of the dual problem (32) is the functional $\mathfrak{F}(\mathbf{u})$. Thus, the constant $C$ might include a factor like $\sum_{i=1}^{2}$ $\left(\|G(x, \cdot)\|_{H^{\frac{1}{2}+p}\left(\Gamma_{i}\right)}+\left\|\partial_{\nu} G(x, \cdot)\right\|_{H^{-\frac{1}{2}+p}\left(\Gamma_{i}\right)}\right)$. Note that this term is finite for all $x \in \mathbb{R}^{2} \backslash \Gamma_{\mathrm{b}}$ and $p \geq 0$. However, because of the singularity of the kernels, it tends to infinity when approaching the boundaries. Thus, also $C$ from (35) might tend to infinity. This effect is even more severe, if we consider functionals that involve derivatives of the kernels, e.g., for the computation of forces and torques using the Maxwell Stress Tensor. Finally, we mention that the regularity assumptions might only hold for smooth surfaces. 
Remark 8 In the linear case, the dual problem to Problem 1 or Problem 4 is the corresponding Bielak-MacCamy coupling [2]. Hence, the same approximation properties hold for the dual problem (see [2]).

Remark 9 Note that similar results as in Theorem 7 and in Remark 7 for the extended Problem 4 can be gained for the interface Problem 1.

\section{Numerical illustration}

To illustrate the theoretical results, we consider for each model problem one example. The description of NURBS geometric entities are obtained by means of the NURBS toolbox included in GeoPDEs, which is implemented in MATLAB (see [17] and [45]). In the same spirit, the required matrices associated to the boundary integral operators are implemented by using, adapting, and supplementing some structures of GeoPDEs. The implementation of the BIOs for arbitrary ansatz functions is performed numerically using standard Gauss-Legendre quadrature for regular contributions and by means of some Duffy-type transformations with a subsequent combination of logarithmic and Gaussian quadrature for the singular parts (see, e.g., [3, Chapter 4.3]). The number of quadrature points on each boundary element is denoted by $N_{\text {Gauss. }}$. In the following, the $\mathcal{H}$ and $\mathcal{H}_{0}$-norm of (10) and (21), respectively, are computed by Gaussian quadrature. However, we replace the noncomputable norm $\|\cdot\|_{H^{-\frac{1}{2}}(\Gamma)}$ by the equivalent norm $\|\cdot\|_{\mathcal{V}}^{2}$ stated in (7). Moreover, we measure the error for the evaluated solution in the BEM domain in the following way. First we define an evaluation path $\Gamma_{\mathrm{e}}$ in the BEM domain. For a certain number $N$ of evaluation points $x_{i} \in \Gamma_{\mathrm{e}}, i=1, \ldots, N, x_{i} \neq x_{j}$, with $i \neq j$, we define the pointwise error as

$$
\text { error }=\max _{i=1, \ldots, N}\left|u^{\mathrm{e}}\left(x_{i}\right)-u_{\ell}^{\mathrm{e}}\left(x_{i}\right)\right| \quad \text { and } \quad \text { error }=\max _{i=1, \ldots, N}\left|u_{\mathrm{b}}\left(x_{i}\right)-u_{\mathrm{b}, \ell}\left(x_{i}\right)\right| .
$$

Here, $u_{\ell}^{\mathrm{e}}$ and $u_{\mathrm{b}, \ell}$ are the discrete evaluations of the corresponding representation formula (4) with the Cauchy data from the corresponding discrete coupling problem. Note that for both problem types the trace has to be calculated with the aid of the jump condition (9c) and (18c), respectively.

In all our experiments, we consider uniform $h$-refinement, for different degrees of B-Splines, starting from the minimal degrees needed to represent the geometry exactly. Increasing the degree of basis functions is called $p$-refinement. Furthermore, note that the number of elements in every $h$-refinement step is calculated by $N_{\mathrm{e}}=$ $\frac{N_{\Omega}}{h^{d}}$, where $N_{\Omega}$ denotes the number of patches and $d$ the dimension of the considered manifold. The element size $h$ is obtained in every refinement step $\ell \in \mathbb{N}$ by $h=\frac{1}{\ell+1}$.

Remark 10 In the following two examples we confirm our results for B-Spline spaces with different polynomial degrees. In particular, we only provide examples with uniform $h$-refinement. Therefore, we do not consider geometries like an L-shaped domain (with a reentrant corner). For such geometries the solution has in general 
singularities, where only a local $h$-refinement of the elements leads to an improved convergence rate (see, e.g., [16]).

\subsection{Single domain}

In the first example, we consider a square domain $\Omega:=(-0.25,0.25)^{2}$ and denote its boundary by $\Gamma$. We parametrize $\Omega$ as a single patch domain using linear B-Spline functions in each parametric direction. It is obvious that Assumption (A5) about the multipatch geometry is satisfied.

Moreover, we consider the interface problem (9) with a linear material tensor $\mathcal{U}:=$ Id, which obviously satisfies (A1), (A2), and $C_{\mathrm{ell}}^{\mathcal{U}}>\frac{1}{4}$. As in [15], we prescribe the exact solutions

$$
u(x)=\left(1-100 x_{1}^{2}-100 x_{2}^{2}\right) e^{-50\left(x_{1}^{2}+x_{2}^{2}\right)}, \quad x=\left(x_{1}, x_{2}\right) \in \Omega,
$$

and

$$
u^{\mathrm{e}}(x)=\log \left(\sqrt{x_{1}^{2}+x_{2}^{2}}\right), \quad x \in \Omega^{\mathrm{e}} .
$$

We calculate the jumps $u_{0}, \phi_{0}$, and the right-hand side $f$ appropriately. Solving the coupled problem using the isogeometric framework, as described in the previous section, yields a discrete solution $\left(u_{\ell}, \phi_{\ell}\right) \in \mathcal{H}_{\ell}:=\mathbb{S}^{0}(\Omega) \times \mathbb{S}^{2}(\Gamma)$. An isogeometric approach for this example is not mandatory since the domain $\Omega$ is standard Cartesian (see, e.g., [15]). However, we want to demonstrate our higher order coupling approach and in particular the super-convergence behavior of this example. Figure 2 shows the solution $u_{\ell} \in \mathbb{S}^{0}(\Omega)$ in the interior domain, as well as the exterior solution $u_{\ell}^{\mathrm{e}}$ in a subset of $\Omega^{\mathrm{e}}$, which we call an evaluation domain $\Omega_{\mathrm{e}}^{\mathrm{e}}:=(-0.5,0.5) \backslash \bar{\Omega}$. The exterior solution $u_{\ell}^{e}$ is obtained from the representation formula $(4)(\kappa=1)$ with the computed Cauchy data $\left(u_{\ell \mid \Gamma}-u_{0}, \phi\right)$ from our discrete solution of the interface problem. Thereby, the degree of the considered B-Spline space for the domain discretization is $p=2$ and its dimension corresponds to an $h$-refinement level $\ell=20$.

As a first numerical experiment, we analyze the convergence of the isogeometric FEM-BEM coupling with respect to the norm $\sqrt{\left\|u-u_{\ell}\right\|_{H^{1}(\Omega)}^{2}+\left\|\phi-\phi_{\ell}\right\|_{\mathcal{V}}^{2}}$, which is equivalent to $\mathcal{H}$-norm in $\Omega$. Since the solution is smooth, the expected order of convergence is equal to the degree of the considered discrete space $\mathcal{H}_{\ell}$, as given in the a priori estimate from Theorem 4. In Fig. 3 we observe the predicted optimal convergence of the method for B-Spline spaces of degree $p=1,2,3,4$.

In the second experiment, we investigate the convergence of the solution in the exterior domain. Note that our exterior solution is smooth. At a first step, we evaluate the solution on an evaluation path $\Gamma_{\mathrm{e}}$, which we define here as the boundary of $(-0.35,0.35)^{2}$. We calculate the error according to (36) with $N=20$ evaluations points. In Fig. 4, we observe a doubling of the convergence rates with respect to the pointwise error, which confirms the theoretical considerations in Remark 7 (see also Remarks 8 and 9).

Furthermore, we want to investigate the dependency of the super-convergence on the position of the evaluation point for a fixed degree $p=3$ of the B-Spline space. For this, we compare the convergence behavior of the exterior solution on 


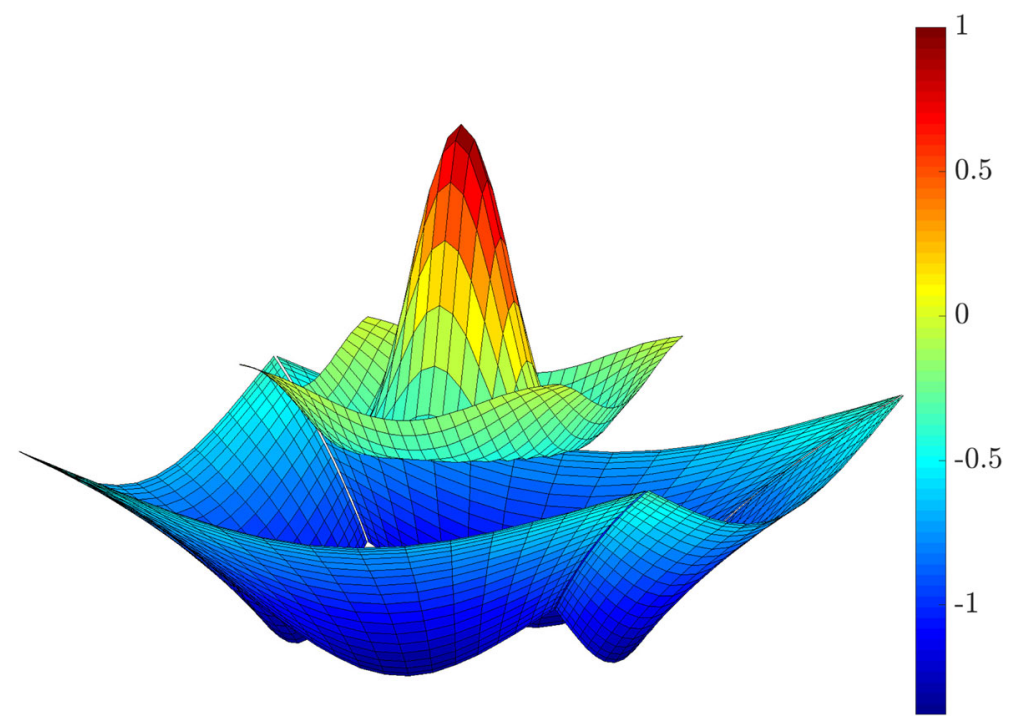

Fig. 2 Solution $\left(u_{\ell}, u_{\ell}^{\mathrm{e}}\right) \in \mathbb{S}^{0}(\Omega) \times \mathbb{S}^{0}\left(\Omega_{\mathrm{e}}^{\mathrm{e}}\right)$ of example in Section 5.1, where we restrict the representation of the exterior solution to $\Omega_{\mathrm{e}}^{\mathrm{e}}:=(-0.5,0.5) \backslash \bar{\Omega}$. The considered B-Spline space corresponds to $\mathbb{S}^{0}(\Omega)$ with degree $p=2$ and an $h$-refinement level $\ell=20$. The exterior solution is obtained by the evaluation of 25 points in each of the 4 exterior patches

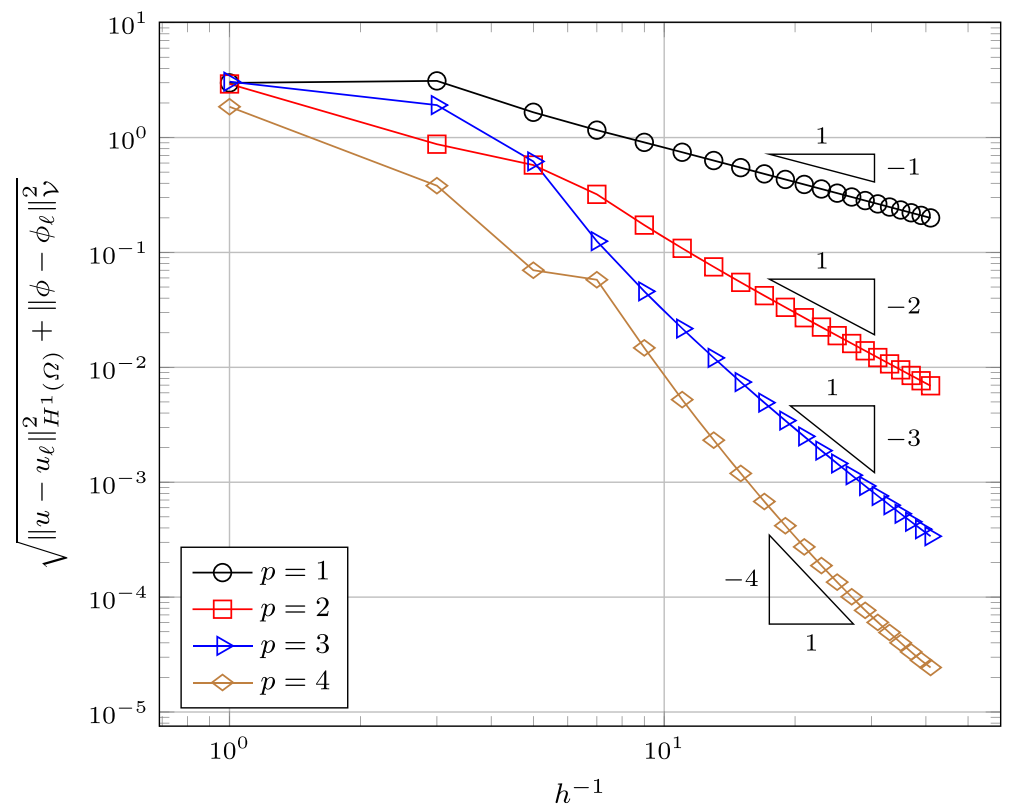

Fig. 3 Convergence of discrete solution $\left(u_{\ell}, \phi_{\ell}\right) \in \mathcal{H}_{\ell}$ to the solution $(u, \phi) \in \mathcal{H}$ for the example in Section 5.1. The considered B-Spline spaces have the degrees $p=1,2,3,4$, respectively, and the error is presented in the norm $\sqrt{\|\cdot\|_{H^{1}(\Omega)}^{2}+\|\cdot\|_{\mathcal{V}}^{2}}$, which is equivalent to the standard $\mathcal{H}=H^{1}(\Omega) \times H^{-\frac{1}{2}}(\Gamma)$ norm 


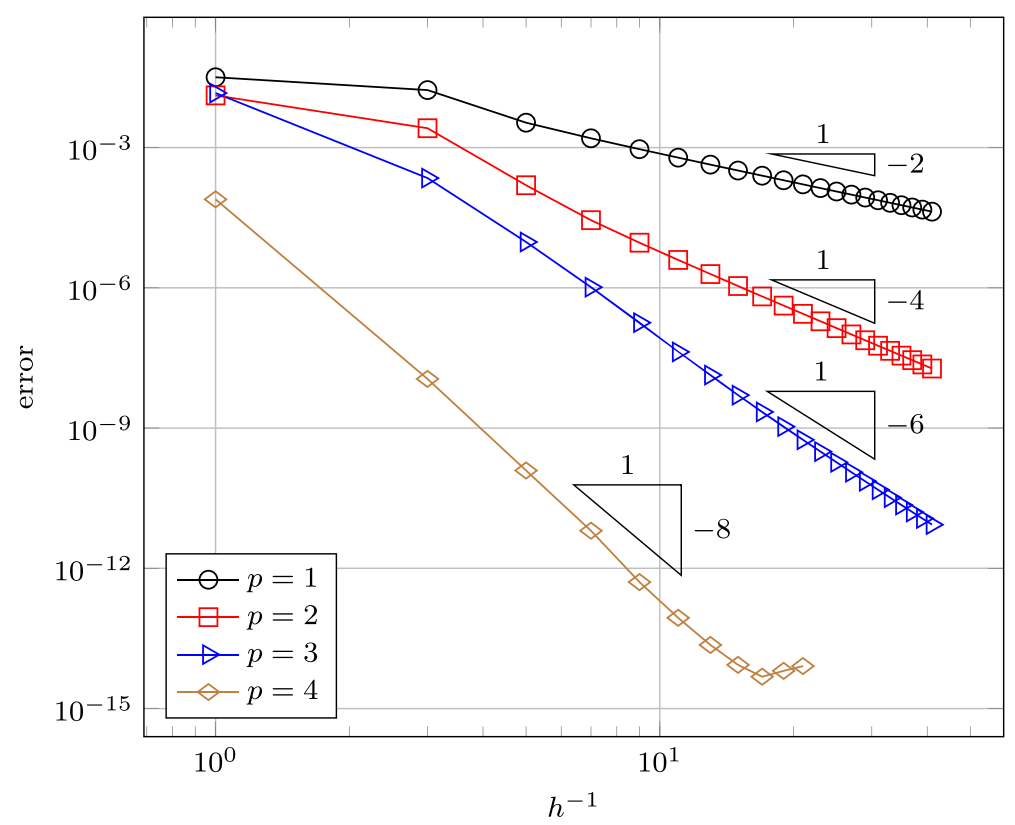

Fig. 4 Convergence of the exterior solution for the example in Section 5.1. The error = $\max _{i=1, \ldots, N}\left|u^{\mathrm{e}}\left(x_{i}\right)-u_{\ell}^{\mathrm{e}}\left(x_{i}\right)\right|$ is calculated with $N=20$ evaluations points on $\Gamma_{\mathrm{e}}$. The considered B-Spline spaces have the degrees $p=1,2,3,4$, respectively. We observe a doubling of the convergence rates. For $p=4$ the error even achieves machine precision

three distinct evaluation paths. We denote the paths by $\Gamma_{\mathrm{e}, 1}, \Gamma_{\mathrm{e}, 2}$, and $\Gamma_{\mathrm{e}, 3}$, which are the boundaries of $(-1,1)^{2},(-0.35,0.35)^{2}$, and $(-0.26,0.26)^{2}$, respectively. For each evaluation path we choose again 20 evaluation points to compute the pointwise error (36). The result is visualized in Fig. 5, where we observe the expected behavior (see Remark 7). In particular, super-convergence is readily observed for the solution on $\Gamma_{\mathrm{e}, 1}$ and $\Gamma_{\mathrm{e}, 2}$. We note that for the error in $\Gamma_{\mathrm{e}, 1}$ we are already at machine precision. Note that the related constant is larger for the solution on $\Gamma_{\mathrm{e}, 2}$, since the path is closer to the interface boundary $\Gamma=\partial \Omega$ with $\Omega:=(-0.25,0.25)^{2}$ than $\Gamma_{\mathrm{e}, 1}$. The same behavior can be observed for the path $\Gamma_{\mathrm{e}, 3}$, which is even closer to $\Gamma$ than $\Gamma_{\mathrm{e}, 2}$. However, the quality of the computation is also deteriorated in the asymptotic area. Additionally, we observe saturation effects for higher refinement levels. This can be improved by increasing the number of Gaussian quadrature points $N_{\text {Gauss }}$ on each boundary element, as it is shown in Fig. 6. However, this in turn is time consuming. With using special extraction techniques, such as the ones developed for 3D in [39], this undesirable effect can be reduced. However, a further investigation is beyond the scope of this work.

\subsection{Multiple domains}

In this second example, we consider the non-symmetric isogeometric FEMBEM coupling for the extended boundary value problem (18) as described in 


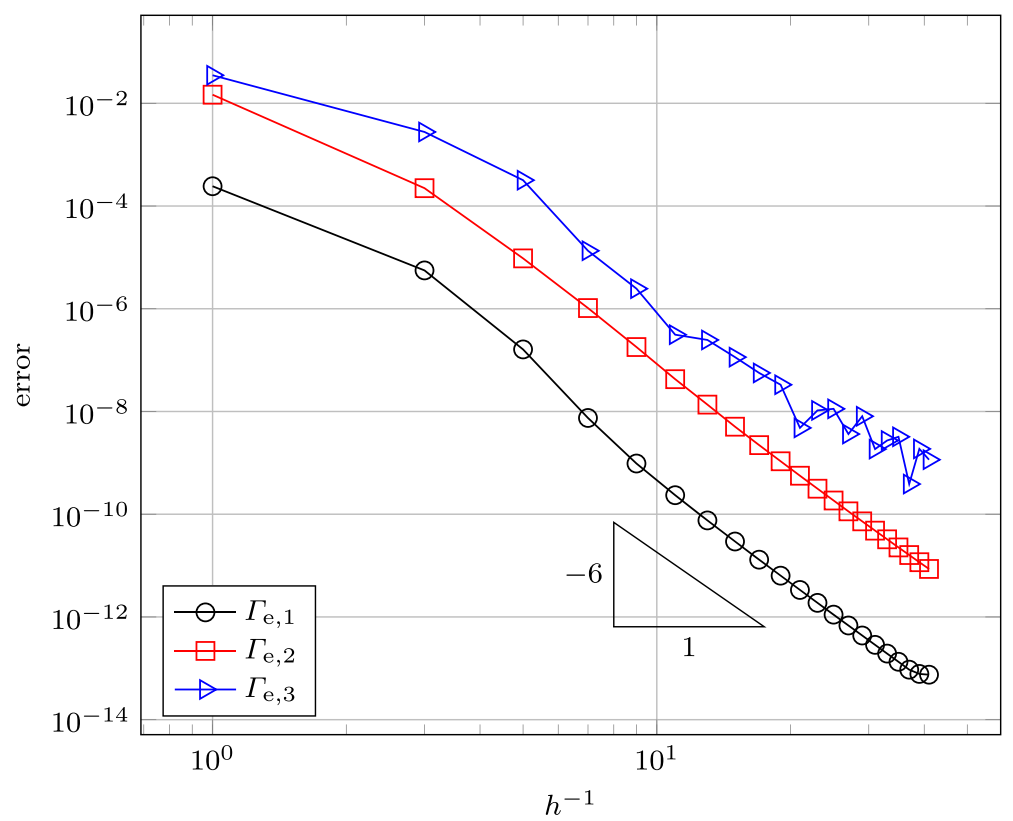

Fig. 5 Dependence of the super-convergence on the evaluation points. The boundary $\Gamma$ of $(-0.25,0.25)^{2}$ is the discretization boundary for the BEM. The $N=20$ evaluations points to calculate the error $=\max _{i=1, \ldots, N}\left|u^{\mathrm{e}}\left(x_{i}\right)-u_{\ell}^{\mathrm{e}}\left(x_{i}\right)\right|$ are on $\Gamma_{\mathrm{e}, 1}, \Gamma_{\mathrm{e}, 2}$, and $\Gamma_{\mathrm{e}, 3}$, which are the boundaries of $(-1,1)^{2},(-0.35,0.35)^{2}$, and $(-0.26,0.26)^{2}$, respectively. We observe the growing constant of the super-convergence constant, which leads to an undesirable saturation for the closest path $\Gamma_{\mathrm{e}, 3}$ with respect to $\Gamma$

Section 4. The topology of the model problem and the notation can be adopted from Fig. 1. However, we consider here a problem domain constructed over circles (see Fig. 7). In particular, if we denote by $B\left(\left(x_{1}, x_{2}\right) ; r\right)$ a circular domain with midpoint $\left(x_{1}, x_{2}\right)$ and radius $r$ we arrive at the following setting: $\Omega_{1}=$ $B((0,0) ; 0.39) \backslash B((0,0) ; 0.1), \Omega_{2}=B((0,0) ; 0.6) \backslash B((0,0) ; 0.4)$, and the thin air gap $\Omega_{\mathrm{b}}=B((0,0) ; 0.4) \backslash B((0,0) ; 0.39)$, which describes in fact three rings. We prescribe the right-hand side $f_{i}$ in $\Omega_{i}$ as

$$
f_{1}\left(x_{1}, x_{2}\right)=0 \quad \text { and } \quad f_{2}\left(x_{1}, x_{2}\right)=100 \sin (\varphi),
$$

where $\varphi$ is the standard angle in a polar coordinate system. In addition, we do not allow jumps, i.e., we set $u_{0, i}=0$ and $\phi_{0, i}=0$. Furthermore, the non-linear material tensor is modeled as follows

$$
\begin{aligned}
\mathcal{U}_{i} \nabla u_{i}:= & g\left(\left|\nabla u_{i}\right|\right) \nabla u_{i}, i=1,2, \quad \text { with } g(t) \\
& = \begin{cases}\frac{h_{\mathrm{c}}}{b_{\mathrm{s}}} & \text { for } t=0, \\
\frac{h_{\mathrm{c}}}{t} \tanh ^{-1}\left(\frac{t}{b_{\mathrm{s}}}\right) & \text { for } 0<t \leq t_{\mathrm{c}}:=b_{\mathrm{s}}-\epsilon, \\
1+\beta \exp (-\alpha t) & \text { for } t>t_{\mathrm{c}},\end{cases}
\end{aligned}
$$

where $h_{\mathrm{c}}$ and $b_{\mathrm{s}}$ depend on the material. Moreover, we choose $\epsilon>0$ arbitrarily such that $g(t)<1$, for all $0<t \leq t_{\mathrm{c}}$, and $\alpha, \beta$ such that $g(t)$ is continuously differentiable 


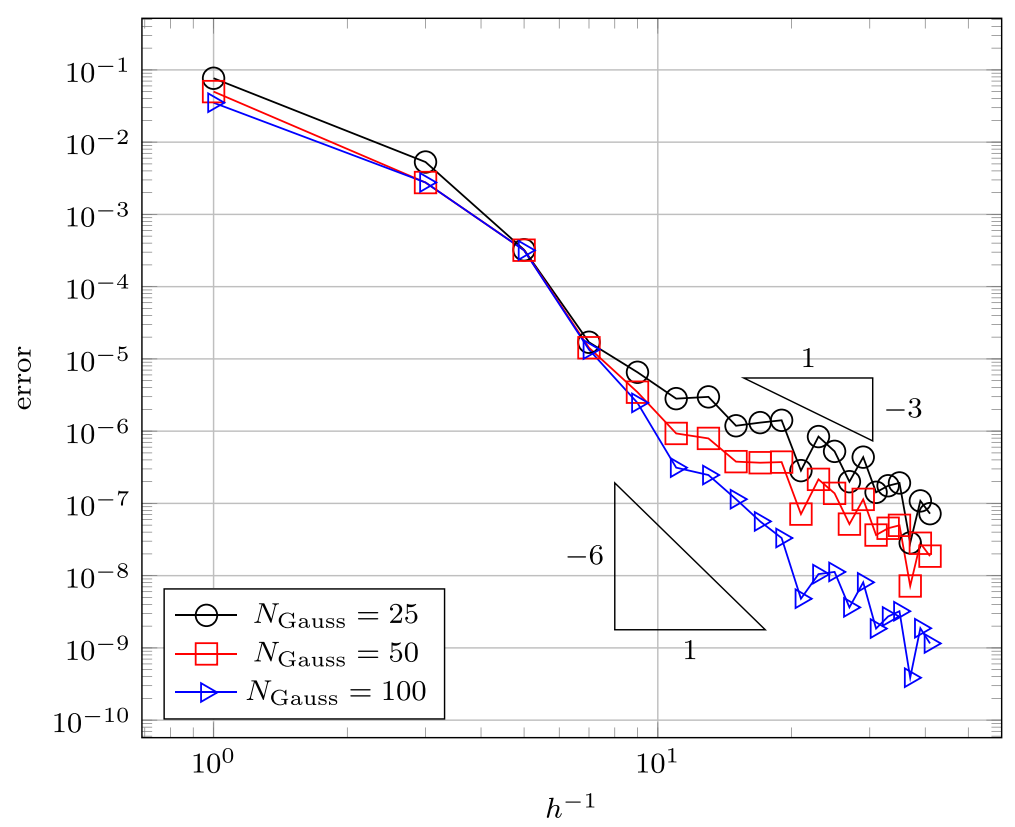

Fig. 6 Dependence of the saturation effect on the number of Gaussian points. The boundary $\Gamma$ of $(-0.25,0.25)^{2}$ is the discretization boundary for the BEM. The evaluations points $N=20$ to calculate error $=\max _{i=1, \ldots, N}\left|u^{\mathrm{e}}\left(x_{i}\right)-u_{\ell}^{\mathrm{e}}\left(x_{i}\right)\right|$ are on $\Gamma_{\mathrm{e}, 3}$ of Fig. 5, i.e., the boundary of $(-0.26,0.26)^{2}$. The number of Gaussian points used for the evaluation as well as the assembling of the matrices are 25,50 , and 100 , respectively. We observe an amelioration of the undesirable saturation with increasing the number of Gaussian points. For $N_{\text {Gauss }}=100$, the expected super-convergence is restored to some extent

for all $t>0$ and strongly monotone. Hence, assumptions (A1) and (A2) are satisfied per construction.

Concretely, the extension coefficients $\alpha$ and $\beta$ are computed as follows:

$$
\alpha=\frac{g^{\prime}\left(t_{\mathrm{c}}\right)}{1-g\left(t_{\mathrm{c}}\right)}, \quad \beta=\left(g\left(t_{\mathrm{c}}\right)-1\right) \exp \left(\alpha t_{\mathrm{c}}\right) .
$$

For this experiment, we choose $\epsilon=10^{-2}, h_{\mathrm{c}}=3 \cdot 10^{-3}$, and $b_{\mathrm{s}}=\frac{3}{2}$. In the following, this particular choice is discussed.

Remark 11 In general, the eigenvalues $\lambda_{1}$ and $\lambda_{2}$ of $C_{\mathrm{ell}}^{\mathcal{U}_{i}}>\frac{1}{4 \lambda_{i}}$ in Theorem 5 and Theorem 6 are not explicitly known. However, for circular domains the values are $\lambda_{1}=\lambda_{2}=1$ (see [32, Section 3] for more details). On the one hand, note that the non-linear material tensor (37) in this particular setting leads to $C_{\mathrm{ell}}^{\mathcal{U}_{i}}=\frac{h_{\mathrm{c}}}{b_{\mathrm{s}}}=2 \cdot 10^{-3}$, which clearly violates the condition $C_{\mathrm{ell}}^{\mathcal{U}_{i}}>\frac{1}{4}$. On the other hand, choosing $h_{\mathrm{c}}$ and $b_{\mathrm{s}}$ such that $\frac{h_{\mathrm{c}}}{b_{\mathrm{s}}}>\frac{1}{4}$ holds is not realistic in the context of electric machines. As discussed in Remark 5, the bound seems to be a theoretical one. Therefore, we prefer to present results with this setting, and test the numerical validity of the method beyond this theoretical restriction. 
Fig. 7 Multipatch

representation (4 patches per domain: the interfaces and boundaries of the patches are highlighted by bold lines) of the example in Section 5.2

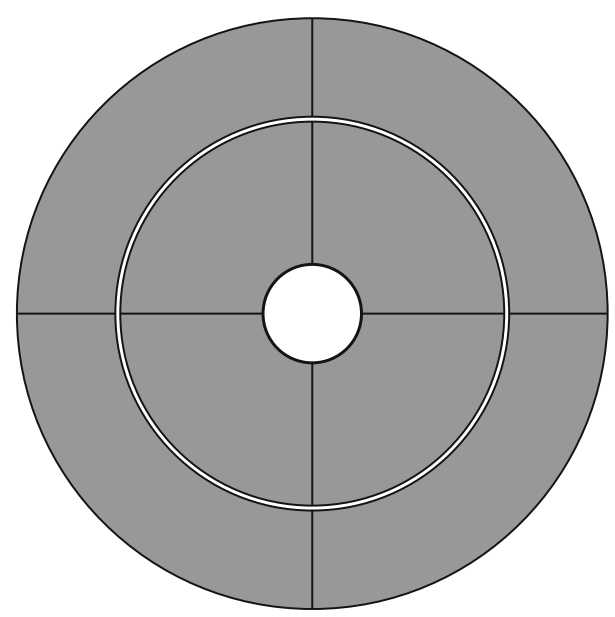

Following the isogeometric approach, we model both domains separately and according to Definition 5 as multipatch domains consisting of four patches (see Fig. 7). Each patch is represented exactly by a NURBS of degree $p=2$ in each parametric direction. Moreover, the Assumption (A5) is obviously satisfied. Note that this model configuration with the circular geometry can be interpreted as a $2 \mathrm{D}$ section of
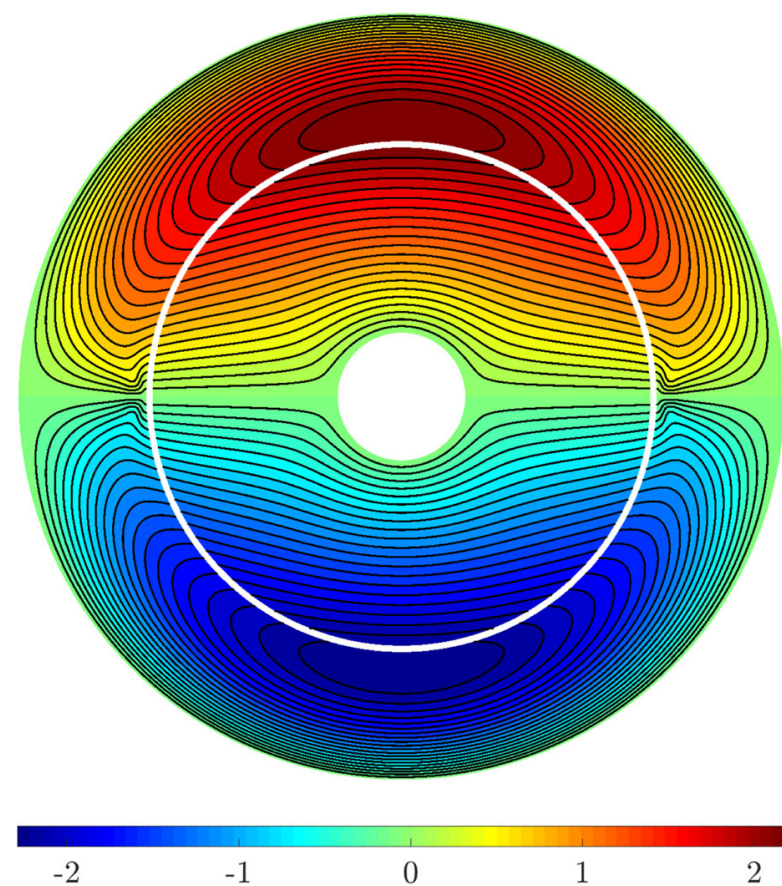

Fig. 8 The solution in the interior domains $\Omega_{1}$ and $\Omega_{2}$ for the electric machine of the example in Section 5.2 with $p=3$ and $\ell=28$. The equipotential lines are the magnetic field lines 
a simplified 2-pole synchronous machine [26, Section 5.2]. This type of applications motivates also the consideration of non-linear operators. In fact, these devices are mainly made of ferromagnetic materials, which are known to be non-linear. In particular, by neglecting anisotropies and hysteresis effects, ferromagnetic materials can be modeled by using non-linear operators of the same type as the ones we considered in Lemma 2 and Lemma 4, and for this example in (37). For more details about this topic see [33] and [36], for instance. Furthermore, we refer to [6] for electrical engineering simulations of electric machines.

In this experiment, the arising non-linear problem is solved by using a standard Picard iteration method. For the stopping criterion, we consider a relative residual error of $10^{-10}$. In our simulation below we need an average of 35 Picard iterations to fulfill the criterion.

The solutions $u_{1}$ and $u_{2}$ in the interior domains $\Omega_{1}$ and $\Omega_{2}$, respectively, are visualized in Fig. 8 for B-Spline spaces of degree $p=3$ and a level of refinement $\ell=28$. In the context of electric machines, $u_{i}, i=1,2$, can be interpreted as the third component of the magnetic vector potential. Note that the equipotential lines, i.e., the continuous black lines in Fig. 8 are the magnetic field lines. The interaction of the magnetic fields stemming from the rotor and the stator in the air gap may induce a mechanical torque. Hence, the rotor, i.e., the interior ring, moves in order to reduce the (spatial) phase shift between both magnetic fields. In particular, the computation

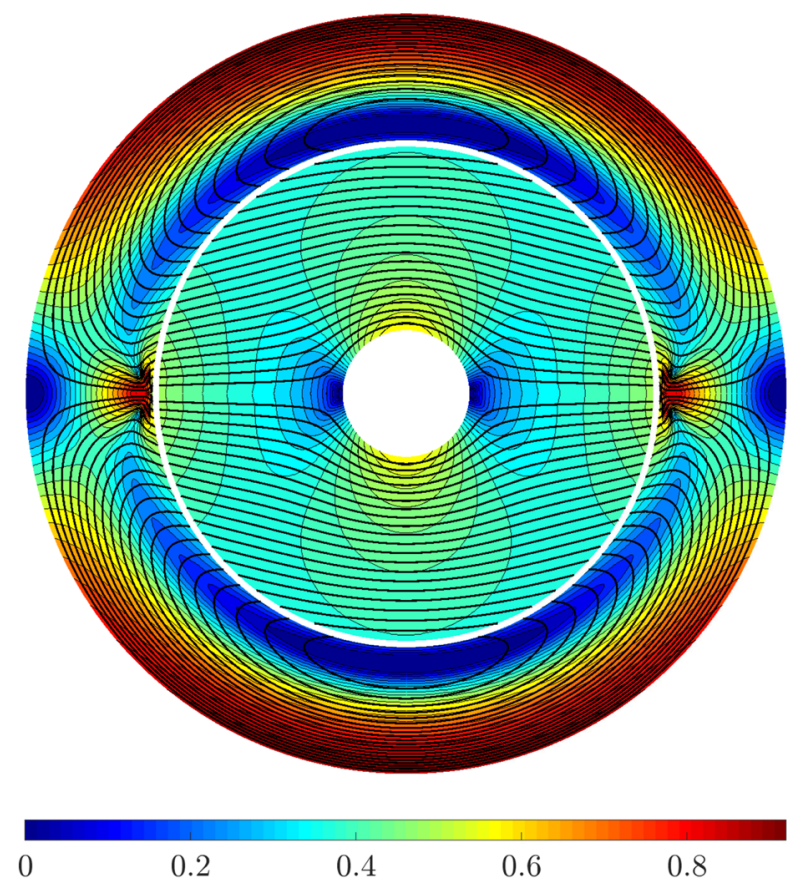

Fig. 9 Saturation effects caused by the non-linear material tensors. The color-bar and the thin lines represent the levels of the magnetic reluctivity, which is given by $g\left(\nabla u_{i}\right)$, for $i=1,2$, and evaluated using the derivatives of the solutions $u_{1}$ and $u_{2}$ obtained with $p=3$ at a refinement level $\ell=28$. The thick equipotential lines show the flow direction of the magnetic field 
of torques and forces involves the computation of the magnetic flux density, which in turn requires the evaluation of the solution and its derivatives in the gap domain, c.f., [27], for instance. Therefore, the super-convergence behavior in the air gap of the machine is of particular interest.

In a post-processing step, we compute the magnetic reluctivity, which is defined as the reciprocal of the magnetic permeability. Formally, it is given by the function $g\left(\left|\nabla u_{i}\right|\right), i=1,2$, in (37), which we evaluate by using the solutions $u_{1}$ and $u_{2}$. Since we are considering non-linear materials, the reluctivity is not constant across the electric machine. This is depicted in Fig. 9. We considered thereby B-Spline spaces of degree $p=3$ and a level of refinement $\ell=28$. Note that the thick black lines are the same as those in Fig. 8, i.e., they represent the equipotential lines of the solutions $u_{1}$ and $u_{2}$.

To verify Remark 7 numerically, we evaluate the solution in the BEM domain $\Omega_{\mathrm{b}}$ on the evaluation path given as the parametrized circle $\partial B((0,0) ; 0.395)$. Note that in this example, the BEM is applied in an interior domain $\Omega_{\mathrm{b}}$. Hence, we use for the evaluation the representation formula (4) with $\kappa=0$ and the complete Cauchy data on $\Gamma_{1}$ and $\Gamma_{2}$, which are available after solving Problem 5 with the jump condition (18c). An analytical solution for our model problem is not known. Hence, to verify the convergence order, we follow a standard procedure: The mesh of the

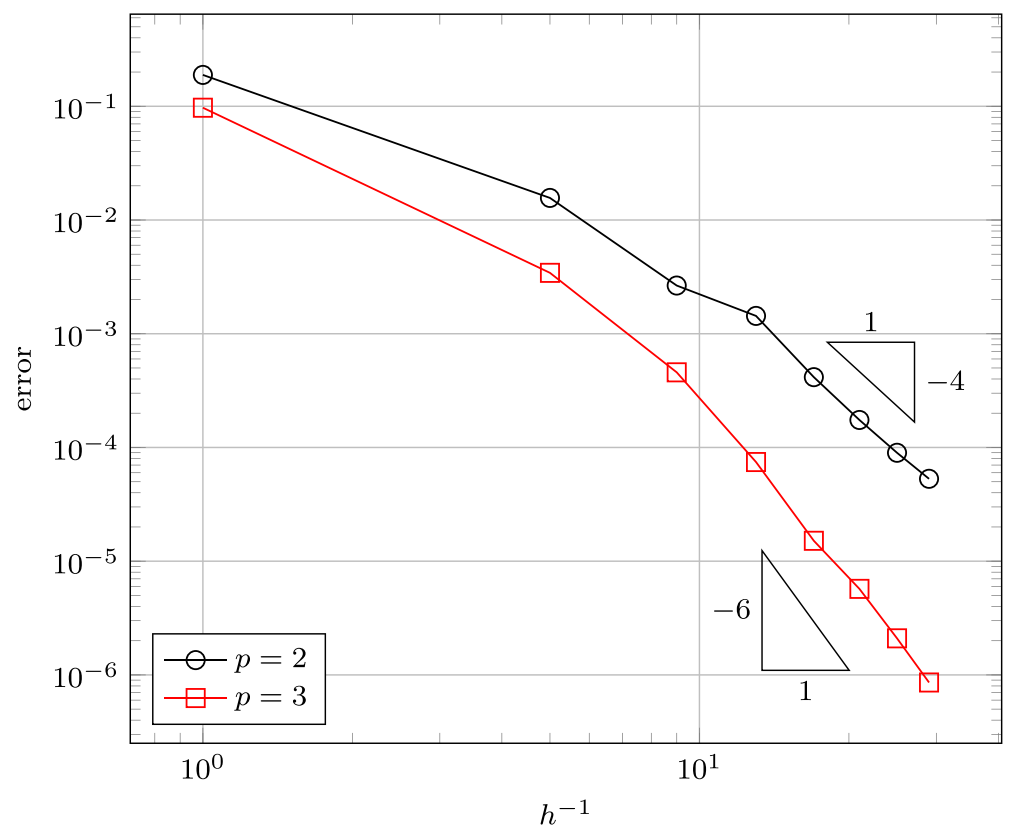

Fig. 10 Convergence of the solution on the evaluation path $\Gamma_{\mathrm{e}}=\partial B((0,0) ; 0.395)$ (circle) in the air gap $\Omega_{\mathrm{b}}$ for the example in Section 5.2. The error $=\max _{i=1, \ldots, N}\left|u^{\mathrm{e}}\left(x_{i}\right)-u_{\ell}^{\mathrm{e}}\left(x_{i}\right)\right|$ is calculated with $N=20$ evaluations points on $\Gamma_{\mathrm{e}}$. As a replacement for the unknown analytical solution we use an Aitken $\Delta^{2}$ extrapolation of a sequence of successively refined discrete solutions 
current solution is successively refined three times and we calculate the corresponding discrete solutions. We apply the Aitkin's $\Delta^{2}$-extrapolation to this sequence of discrete solutions and this extrapolated value is the reference solution $u^{\mathrm{e}}\left(x_{i}\right)$ for the error $=\max _{i=1, \ldots, N}\left|u^{\mathrm{e}}\left(x_{i}\right)-u_{\ell}^{\mathrm{e}}\left(x_{i}\right)\right|$ calculated from $N=20$ evaluations points. This error is visualized in Fig. 10 for ansatz spaces of degree $p=2$ and $p=3$, where we observe an amelioration of the convergence rates. Note that this amelioration depends on the quality of the numerical integration, as shown in Fig. 6 for the example of Section 5.1. For this example, noticeable amelioration of the convergence rates were only observable for a high number of Gaussian quadrature points. In this case, $N_{\text {Gauss }}=400$ points were considered for the assembling of the BEM matrices, which is very time consuming. The dominance of the quadrature error for this type of evaluations can however be tackled, as mentioned in the previous section, by using special extraction techniques. Moreover, efficient assembly of the BEM matrices based on B-Spline tailored quadrature rules, as given in [1], together with suitable compression methods (see e.g., [12]), would accelerate the computation considerably. However, this investigation is beyond the scope of this work.

\section{Conclusions}

The non-symmetric FEM-BEM coupling in the isogeometric context for simulating practical problems with complex geometries turns out to be a promising alternative to classical approaches. A transformation to an integral formulation allows a problem in a domain to be reduced to its boundary, where the BEM can be applied. For exterior problems there is no need to truncate the unbounded domain, and for simulating thin gaps there is no need for a complicated remeshing. In both cases numerical errors can be avoided. Thanks to the definition of B-Splines, $h$ - and $p$-refinements are applied in a straightforward manner. Furthermore, multiple domain modeling can be done independently. This is particularly advantageous if we consider moving or deforming geometries. A classical transmission and a multiple domain problem with parts of non-linear material are considered. Obviously, FEM is applied to the non-linear areas, whereas BEM is exclusively used for the linear problem. For both model problems, well-posedness for the continuous and discrete problem, and quasi-optimality and convergence rates for the numerical approximation are mathematically analyzed in the isogeometric framework. Furthermore, we show an improvement of the convergence behavior, if we consider the error in functionals of the solution. This is motivated by a practice-oriented application such as electric machines. Here the computation of torques are a central task and involve the evaluation of some derivatives of the solution in the BEM domain. We observe for both model applications this super-convergence, which confirms the theory. Future extensions of the method may include the consideration of parabolic-elliptic problems and a rigorous analysis of the coupling for curl curl-type equations in 3D.

Funding Open Access funding enabled and organized by Projekt DEAL. The research of Mehdi Elasmi was supported in parts by the Excellence Initiative of the German Federal and State Governments and the Graduate School of Computational Engineering at TU Darmstadt. 
Open Access This article is licensed under a Creative Commons Attribution 4.0 International License, which permits use, sharing, adaptation, distribution and reproduction in any medium or format, as long as you give appropriate credit to the original author(s) and the source, provide a link to the Creative Commons licence, and indicate if changes were made. The images or other third party material in this article are included in the article's Creative Commons licence, unless indicated otherwise in a credit line to the material. If material is not included in the article's Creative Commons licence and your intended use is not permitted by statutory regulation or exceeds the permitted use, you will need to obtain permission directly from the copyright holder. To view a copy of this licence, visit http://creativecommons.org/licenses/by/4.0/.

\section{References}

1. Aimi, A., Calabro, F., Diligenti, M., Sampoli, M.L., Sangalli, G., Sestini, A.: Efficient assembly based on B-spline tailored quadrature rules for the IgA-SGBEM. Comput. Methods Appl. Mech. Eng. 331, 327-342 (2018)

2. Aurada, M., Feischl, M., Führer, T., Karkulik, M., Melenk, J.M., Praetorius, D.: Classical FEM-BEM coupling methods: nonlinearities, well-posedness, and adaptivity. Comput. Mech. 51(4), 399-419 (2013)

3. Bantle, A.: On high-order NURBS-based boundary element methods in two dimensions-numerical integration and implementation. Ph.D. thesis, Fakultät für Mathematik und Wirtschaftswissenschaften, Universität Ulm (2015)

4. Bazilevs, Y., Beirão da Veiga, L., Cottrell, J.A., Hughes, T.J.R., Sangalli, G.: Isogeometric analysis: approximation, stability and error estimates for h-refined meshes. Mathematical Models and Methods in Applied Sciences 16(07), 1031-1090 (2006). https://doi.org/10.1142/S0218202506001455

5. Bielak, J., MacCamy, R.C.: An exterior interface problem in two-dimensional elastodynamics. Q. Appl. Math. 41(1), 143-159 (1983)

6. Bontinck, Z., Corno, J., Schöps, S., De Gersem, H.: Isogeometric analysis and harmonic statorrotor coupling for simulating electric machines. Comput. Methods Appl. Mech. Eng. 334, 40-55 (2018). https://doi.org/10.1016/j.cma.2018.01.047. https://www.sciencedirect.com/science/article/pii/ S0045782518300549

7. Buffa, A., Dölz, J., Kurz, S., Schöps, S., Vázquez, R., Wolf, F.: Multipatch approximation of the de Rham sequence and its traces in isogeometric analysis. Numer. Math. 144(1), 201-236 (2020)

8. Corno, J., de Falco, C., De Gersem, H., Schöps, S.: Isogeometric simulation of Lorentz detuning in superconducting accelerator cavities. Comput. Phys. Commun. 201, 1-7 (2016)

9. Costabel, M.: Boundary integral operators on Lipschitz domains: elementary results. SIAM J. Math. Anal. 19(3), 613-626 (1988)

10. Costabel, M.: A symmetric method for the coupling of finite elements and boundary elements. The Mathematics of Finite Elements and Applications VI(Uxbridge, 1987), 281-288 (1988)

11. Cottrell, J.A., Hughes, T.J., Bazilevs, Y.: Isogeometric analysis: toward integration of CAD and FEA. Wiley (2009)

12. Dölz, J., Kurz, S., Schöps, S., Wolf, F.: Isogeometric boundary elements in electromagnetism: Rigorous analysis, fast methods, and examples. SIAM J. Sci. Comput. 41(5), B983-B1010 (2019)

13. Egger, H., Erath, C., Schorr, R.: On the nonsymmetric coupling method for parabolic-elliptic interface problems. SIAM J. Numer. Anal. 56(6), 3510-3533 (2018). https://doi.org/10.1137/17M1158276

14. Erath, C.: Coupling of the finite volume element method and the boundary element method: an a priori convergence result. SIAM J. Numer. Anal. 50(2), 574-594 (2012). https://doi.org/10.1137/ 110833944

15. Erath, C., Of, G., Sayas, F.: A non-symmetric coupling of the finite volume method and the boundary element method. Numer. Math. 135(3), 895-922 (2017)

16. Erath, C., Schorr, R.: An adaptive nonsymmetric finite volume and boundary element coupling method for a fluid mechanics interface problem. SIAM J. Sci. Comput. 39(3), A741-A760 (2017). https://doi.org/10.1137/16M1076721

17. de Falco, C., Reali, A., Vázquez, R.: GeoPDEs: a research tool for isogeometric analysis of PDEs. Adv. Eng. Softw. 42(12), 1020-1034 (2011)

18. Feischl, M., Gantner, G., Haberl, A., Praetorius, D.: Adaptive 2d iga boundary element methods. Engineering Analysis with Boundary Elements 62, 141-153 (2016). https://doi.org/10.1016/j. enganabound.2015.10.003. http://www.sciencedirect.com/science/article/pii/S0955799715002155 
19. Feischl, M., Gantner, G., Praetorius, D.: Reliable and efficient a posteriori error estimation for adaptive iga boundary element methods for weakly-singular integral equations. Comput. Methods Appl. Mech. Eng. 290, 362-386 (2015). https://doi.org/10.1016/j.cma.2015.03.013. http://www.sciencedirect.com/ science/article/pii/S0045782515001231

20. Führer, T., Gantner, G., Praetorius, D., Schimanko, S.: Optimal additive schwarz preconditioning for adaptive $2 \mathrm{~d}$ iga boundary element methods. Comput. Methods Appl. Mech. Eng. 351, 571-598 (2019). https://doi.org/10.1016/j.cma.2019.03.038. http://www.sciencedirect.com/science/article/pii/ S0045782519301689

21. Gantner, G.: Adaptive isogeometrische bem. Master's thesis, Vienna University of Technology. https:// resolver.obvsg.at/urn:nbn:at:at-ubtuw:1-64362 (2014)

22. Gantner, G.: Optimal adaptivity for splines in finite and boundary element methods. Ph.D. thesis, Vienna University of Technology. https://resolver.obvsg.at/urn:nbn:at:at-ubtuw:1-105591 (2017)

23. Harbrecht, H., Randrianarivony, M.: From computer aided design to wavelet bem. Comput. Methods Appl. Mech. Eng. 13(2), 69-82 (2009). https://doi.org/10.1007/s00791-009-0129-1

24. Hughes, T., Cottrell, J.: Isogeometric analysis: CAD, finite elements, NURBS, exact geometry and mesh refinement. Comput. Methods Appl. Mech. Eng. 194(39), 4135-4195 (2005). https://doi.org/10. 1016/j.cma.2004.10.008. http://www.sciencedirect.com/science/article/pii/S0045782504005171

25. Johnson, C., Nédélec, J.C.: On the coupling of boundary integral and finite element methods. Mathematics of Computation 35, 1063-1079 (1980)

26. Kurz, S.: Die numerische Behandlung elektromechanischer Systeme mit Hilfe der Kopplung der Methode der finiten Elemente und der Randelementmethode. VDI-Verlag (1998)

27. Kurz, S., Fetzer, J., Kube, T., Lehner, G., Rucker, W.M.: BEM-FEM coupling in electromechanics: A 2-D watch stepping motor driven by a thin wire coil. Appl. Comput. Electromagn. Soc. J. 12, 135-139 (1997)

28. MacCamy, R.C., Suri, M.: A time-dependent interface problem for two-dimensional eddy currents. Quart. Appl. Math. 44, 675-690 (1987)

29. Marussig, B., Zechner, J., Beer, G., Fries, T.P.: Fast isogeometric boundary element method based on independent field approximation. Comput. Methods Appl. Mech. Eng. 284, 458-488 (2015). https://doi.org/10.1016/j.cma.2014.09.035. http://www.sciencedirect.com/science/article/pii/ S0045782514003582. Isogeometric Analysis Special Issue

30. McLean, W.: Strongly Elliptic Systems and Boundary Integral Equations. Cambridge University Press, Cambridge (2000)

31. Of, G., Steinbach, O.: Is the one-equation coupling of finite and boundary element methods always stable? ZAMM-Journal of Applied Mathematics and Mechanics/Zeitschrift fü,r Angewandte Mathematik und Mechanik 93(6-7), 476-484 (2013)

32. Of, G., Steinbach, O.: On the ellipticity of coupled finite element and one-equation boundary element methods for boundary value problems. Numer. Math. 127(3), 567-593 (2014)

33. Pechstein, C.: Multigrid-newton-methods for nonlinear magnetostatic problems. Master's thesis, Johannes Kepler University of Linz, Institute of Computational Mathematics, Linz. http://www.numa. uni-linz.ac.at/Teaching/Diplom/Finished/pechstein (2004)

34. Piegl, L., Tiller, W.: The NURBS book. Springer Science \& Business Media (2012)

35. Politis, C., Ginnis, A.I., Kaklis, P.D., Belibassakis, K., Feurer, C.: An isogeometric bem for exterior potential-flow problems in the plane. In: 2009 SIAM/ACM Joint Conference on Geometric and Physical Modeling, SPM '09, pp. 349-354. Association for Computing Machinery, New York (2009). https://doi.org/10.1145/1629255.1629302

36. Römer, U.: Numerical approximation of the magnetoquasistatic model with uncertainties and its application to magnet design. Ph.D. thesis, Institut für Theorie Elektromagnetischer Felder, Technische Universität Darmstadt (2015)

37. Sauter, S.A., Schwab, C.: Boundary element methods. In: Boundary Element Methods, pp. 183-287. Springer (2010)

38. Sayas, F.: The validity of Johnson-Nédélec's BEM-FEM coupling on polygonal interfaces. SIAM J. Numer. Anal. 47(5), 3451-3463 (2009)

39. Schwab, C., Wendland, W.: On the extraction technique in boundary integral equations. Mathematics of Computation 68(225), 91-122 (1999)

40. Scott, M., Simpson, R., Evans, J., Lipton, S., Bordas, S., Hughes, T., Sederberg, T.: Isogeometric boundary element analysis using unstructured t-splines. Comput. Methods Appl. Mech. Eng. 254, 197-221 (2013). https://doi.org/10.1016/j.cma.2012.11.001. http://www.sciencedirect.com/science/ article/pii/S0045782512003386 
41. Steinbach, O.: Numerical approximation methods for elliptic boundary value problems: finite and boundary elements. Springer Science \& Business Media (2007)

42. Steinbach, O.: A note on the stable one-equation coupling of finite and boundary elements. SIAM J. Numer. Anal. 49(4), 1521-1531 (2011)

43. Steinbach, O.: On the stability of the non-symmetric BEM/FEM coupling in linear elasticity. Computational Mechanics 51(4), 421-430 (2013)

44. Beirão da Veiga, L., Buffa, A., Sangalli, G., Vázquez, R.: Mathematical analysis of variational isogeometric methods. Acta Numerica 23, 157-287 (2014)

45. Vázquez, R.: A new design for the implementation of isogeometric analysis in octave and matlab: Geopdes 3.0. Computers \& Mathematics with Applications 72(3), 523-554 (2016). https://doi.org/10. 1016/j.camwa.2016.05.010. https://www.sciencedirect.com/science/article/pii/S0898122116302681

46. Zeidler, E.: Nonlinear functional analysis and its applications II/B: Nonlinear monotone operators. Springer (1986)

Publisher's note Springer Nature remains neutral with regard to jurisdictional claims in published maps and institutional affiliations.

\section{Affiliations}

\section{Mehdi Elasmi $^{1}$ (D) . Christoph Erath ${ }^{2}$. Stefan Kurz ${ }^{1}$}

Christoph Erath

christoph.erath@ph-vorarlberg.ac.at

Stefan Kurz

kurz@gsc.tu-darmstadt.de

1 Technische Universität Darmstadt, Institute for Accelerator Science and Electromagnetic Fields, Schlossgartenstraße 8, 64289 Darmstadt, Germany

2 University College of Teacher Education Vorarlberg (PH Vorarlberg), Liechtensteinerstraße 33-37, 6800 Feldkirch, Austria 